\title{
Exogenous Glucocorticoids Decrease Subgenual Cingulate Activity Evoked by Sadness
}

\author{
Keith D Sudheimer*,I,2, James L Abelson', Stephan F Taylor', Brian Martis', Robert C Welsh', \\ Christine Warner', Mira Samet', Andrea Manduzzi' and Israel Liberzon' \\ 'Department of Psychiatry, University of Michigan, Ann Arbor, MI, USA; '2Department of Psychiatry and Behavioral Sciences, Stanford University, \\ Stanford, CA, USA
}

\begin{abstract}
The glucocorticoid hormone cortisol is known to have wide-ranging effects on a variety of physiological systems, including the morphology and physiology of the amygdala and hippocampus. Disruptions of cortisol regulation and signaling are also linked with psychiatric disorders involving emotional disturbances. Although there is much evidence to suggest a relationship between cortisol signaling and the brain physiology underlying emotion, few studies have attempted to test for direct effects of cortisol on the neurophysiology of emotion. We administered exogenous synthetic cortisol (hydrocortisone, HCT) using two different dosing regimens ( $25 \mathrm{mg} /$ day over 4 days, $100 \mathrm{mg}$ single dose), in a double-blind placebo-controlled functional magnetic resonance imaging (fMRI) study. During fMRI scanning, healthy subjects viewed images designed to induce happy, sad, and neutral emotional states. Subjective emotional reactions were collected for each experimental stimulus after $\mathrm{MRI}$ scanning. Mood ratings were also collected throughout the 4 days of the study. Both dose regimens of HCT resulted in decreased subgenual cingulate activation during sadness conditions. The $25 \mathrm{mg} /$ day regimen also resulted in higher arousal ratings of sad stimuli. No effects of HCT were observed on any mood ratings. Few reliable effects of HCT were observed on brain activity patterns or subjective emotional responses to stimuli that were not sad. The inhibitory effects of cortisol on sadness-induced subgenual cingulate activity may have critical relevance to the pathophysiology of major depression, as both subgenual hyperactivity and decreased sensitivity to cortisol signaling have been documented in patients with depression. Neuropsychopharmacology (2013) 38, 826-845; doi:I0.1038/npp.20 I2.249; published online 16 January 2013
\end{abstract}

Keywords: cortisol; emotion; fMRl; glucocorticoids; corticosteroids; subgenual

\section{INTRODUCTION}

The glucocorticoid hormone cortisol has wide-ranging effects on many physiological systems, including the brain. When exogenous glucocorticoids are administered for behavioral studies (Buchanan and Lovallo, 2001; Reuter, 2002; Wirth et al, 2011) or given therapeutically for controlling inflammatory processes, they can induce substantial emotional changes, including depression and mania (Brown et al, 2004; Ling et al, 1981; Wada et al, 2001; Wada et al, 2000).

Although it is clear that glucocorticoids can influence emotion, the mechanism of these effects is unclear. There is evidence to suggest that glucocorticoids may be exerting these effects by directly influencing the activity of brain regions involved in emotion processing. Cortisol, crosses the blood-brain barrier with high efficiency (Pariante et al, 2004) and has receptors in brain regions implicated in

*Correspondence: Dr KD Sudheimer, Department of Psychiatry and Behavioral Sciences, Stanford University, 40I Quarry Road, Stanford, CA 94304, USA. E-mail: ksudheim@stanford.edu

Received 18 July 2012; revised 2 November 2012; accepted 28 November 2012; accepted article preview online 3 December 2012 emotion processes, including the amygdala, frontal lobe, and temporal lobe (Sarrieau et al, 1986; Watzka et al, 2000a, b). Glucocorticoids have also been shown to have effects on brain activation in the medial temporal lobe (De Quervain et al., 2003), amygdala (Henckens et al, 2010; Lovallo et al, 2010), and hippocampus (Abercrombie et al, 2011). In addition, previous studies have also demonstrated that glucocorticoids affect the morphology and physiology of neurons in brain regions associated with emotional behaviors, including the hippocampus, amygdala, ventral tegmentum, and prefrontal cortex (Brown et al, 2008; Cho and Little, 1999; De Kloet et al, 1998; Karst et al, 2002; Mitra and Sapolsky, 2008; Sapolsky, 2000; Wellman, 2001; Woolley et al, 1990).

The influence of glucocorticoids on emotion-related brain activity could be critically relevant for understanding the pathophysiology of emotion disturbances in psychiatric conditions. Major depressive disorder (MDD) in particular has been associated with changes in glucocorticoid signaling. Reduced glucocorticoid receptor mRNA expression (Webster et al, 2002) has been shown in MDD patients. Similarly, reduced glucocorticoid sensitivity in MDD predicts worse clinical outcomes in patients (Greden et al, 1980; Zobel et al, 2001; Zobel et al, 1999). This suggests that 
glucocorticoid signaling may be as critical as glucocorticoid secretion in MDD.

\section{Objectives and Hypotheses}

The broad objective of this study was to test how exogenous glucocorticoids affect brain activity patterns elicited by specific emotions. As little is known about the normal effects of glucocorticoids on human brain activity, we tested for these effects in young healthy subjects. However, as both glucocorticoid signaling abnormalities and abnormal brain activity patterns are well documented in major depression, we tested for glucocorticoid effects on specific brain regions and emotional processes impacted by MDD. These included the responses of the subgenual cingulate cortex, amygdala, and ventral medial prefrontal cortex to both happy and sad emotional stimuli.

We tested the effects of two different dose regimens of orally administered hydrocortisone (HCT) on brain activity elicited by sad, happy, and neutral emotions. We also tested the effects of HCT on the subjective emotional reactions to stimuli and on mood ratings. We hypothesized HCT would exert effects on sadness-evoked activity in the subgenual cingulate cortex, amygdala, and ventral medial prefrontal cortex. We also hypothesized that subjects would experience emotional changes that would mimic very small-scale depressive symptoms, namely that HCT would increase sadness and decrease happiness reactions to emotional stimuli, and that subjects would experience an increase in sad mood over the course of a 4-day exposure to HCT.

\section{MATERIALS AND METHODS}

\section{Subjects}

A total of 61 healthy ( 31 male, 30 female) subjects, ages 1830 years, were recruited from the community via advertising and signed a local internal review board-approved informed consent document. Exclusion criteria consisted of a history of endocrine disorders, head injury, psychiatric or neurological disorders, current medication use, oral contraceptive use, recent major surgery, a history of traumatic life events, current illicit drug use, smoking, left handedness, and current exposure to self-reported excessive psychological stress, meeting MINI International Neuropsychiatric Interview (Sheehan et al, 1998) criteria for axis-1 psychiatric disorders, scoring more than 7 points on the Beck Depression inventory-II, or testing positive on urine-based drug or pregnancy tests. For female subjects, functional magnetic resonance imaging (fMRI) scanning was scheduled to coincide with the luteal phase of their menstrual cycle based on the last onset of menses.

\section{Design}

This study used a double-blind placebo-controlled betweengroups design. We compared (1) measures of mood (2) subjective reactions to emotional stimuli, and (3) brain activation patterns elicited by happy, sad, and neutral stimuli between a control group receiving placebo and two groups each receiving one of two different dose regimens of HCT.

\section{Matching and Randomization}

Subjects were matched into triplets by age, weight, and gender. All three members of a triplet were required to be within 5 years of each other in age, $4.5 \mathrm{~kg}$ of each other in weight and of the same gender. Each member of a triplet was then randomly assigned into one of the three groups. Subjects received placebo (P), a single dose (SD) of $100 \mathrm{mg}$ oral HCT, given $2 \mathrm{~h}$ before fMRI scanning, or an extended dose (ED) regimen of $25 \mathrm{mg}$ daily over 4 days, given on a twice-daily schedule. Placebos were also administered to the $\mathrm{SD}$ and ED groups at various time points so that all the groups received an identical number of pills at identical times of day. The SD regimen was designed to test acute effects of cortisol by insuring high levels at the time of scanning. The ED regimen was designed to test the effects of persistent elevations, spread over 4 days. The last HCT dose given to the ED group was administered $8 \mathrm{~h}$ before scanning to allow adequate time for metabolism of the last dose. This procedure prevented both acute effects and extended exposure effects of HCT occurring at the same time in the ED group. The matching procedure successfully produced groups of similar age, weight, and gender distributions ( $\mathrm{P}$ : age $\quad$ (years $)=21.8 \quad(0.6)$, weight $\quad(\mathrm{kg})=69.5 \quad(2.8)$, gender $=10 \mathrm{M} / 10 \mathrm{~F} ; \mathrm{SD}$ : age $=21.5 \quad(0.7)$, weight $=69.1$ (2.7), gender $10 \mathrm{M} / 11 \mathrm{~F}$; age $=21.7(0.6)$, weight $=67.3(4.0)$, gender $10 \mathrm{M} / 10 \mathrm{~F})$. One extra subject was recruited to supplement one of the triplets after a single subject only completed half of the fMRI scanning session.

\section{fMRI Task Design}

During scanning subjects passively viewed happy, sad, and neutral stimuli taken from the International Affective Picture System (IAPS) and a collection of emotional face images (Gur et al, 2002). Individual stimuli were presented for $6 \mathrm{~s}$ at a time within a block of similar stimuli that lasted 18,24 , or $30 \mathrm{~s}$. A block of stimuli consisted of only one of the following stimuli types: happy faces, sad faces, neutral faces, happy IAPS, sad IAPS, and neutral IAPS. Stimulus emotion (sad, happy, or neutral) or type (face or IAPS) were not mixed within a block. Each block was temporally flanked by $12 \mathrm{~s}$ of a fixation cross. Twelve blocks were then grouped into runs that lasted $6 \mathrm{~min}$ and $27 \mathrm{~s}$ each. Block order within a run, and image order within a block were pseudo-randomized to control for order effects of particular emotions, stimulus types (IAPS or face), gender of face, and the order/frequency, in which an individual model's face was presented. Each run contained identical and proportional representation of each emotion condition. Subjects were instructed to 'Look at each picture, and feel whatever you feel', and to perform a button press (right index finger) at the onset of each picture or fixation cross. The purpose of the button press was to verify task compliance.

\section{Measurement of Mood States and Emotional Reactions to Stimuli}

Ratings of mood states were collected outside of the laboratory using the expanded form of the positive and negative affect schedule (PANAS-X) (Watson et al, 1988), administered four times per day (0800 hours, 1200 hours, 
1600 hours, and 2000 hours.) over 4 days. The PANAS-X consists of 60 adjectives that reflect current subjective emotional experience. It also contains 11 subscales comprised of groups of related adjectives: attentiveness, fatigue, fear, guilt, hostility, joviality, sadness, self-assuredness, serenity, shyness, and surprise (Watson and Clark, 1994).

The magnitude of happiness, sadness, neutrality, valence, and arousal experienced in reaction to each stimulus used in the fMRI session was also collected using a series of Likert scales in a separate ratings session, which immediately followed scanning. The valence and arousal rating scales used the self-assessment manikin system (Lang et al, 1999). Subjects were instructed to rate how the picture 'Actually makes you feel'.

\section{Measurement of Cortisol}

Nine saliva samples were collected via salivettes from each subject. Samples were collected every $20 \mathrm{~min}$ for $2 \mathrm{~h}$ before fMRI scanning (1400 hours-1600 hours) and then immediately after completion of scanning (1730 hours) and $20 \mathrm{~min}$ later (1750 hours). Cortisol was assayed using the standard direct, non-extraction, Coat-A-Count radioimmunoassay kit (Diagnostic Products Corporation, Los Angeles, CA).

\section{Statistical Analysis of Emotion and Cortisol Measures}

Emotional reactions to experimental stimuli and PANAS-X mood ratings were both analyzed using multivariate ANOVA models, with gender and group as betweensubjects factors. For the emotional reactions to experimental stimuli, we included ratings of the happiness, sadness, valence, and arousal in the multivariate model. For the PANAS-X mood ratings, each of the 11 subscales were used as dependent variables sampled repeatedly over the 4 days of the experiment. The sadness subscale was hypothesized (a priori) to be sensitive to cortisol and predicted to increase over the 4 days in the ED group. For the emotional reactions to experimental stimuli, sadness evoked by sad stimuli, happiness evoked by happy stimuli and arousal evoked by both sad and happy stimuli were each hypothesized to be sensitive to cortisol.

Cortisol assay results were log transformed (for normality) and analyzed using a repeated measures ANOVA model with gender and group as between-subjects factors and time as the repeated measure. Average cortisol during scanning (average of pre- and post-scan salivary cortisol) and average peak cortisol were also calculated and group differences were tested using separate univariate ANOVA models with gender and group as between-subjects factors.

\section{fMRI Acquisition and Analysis}

MRI imaging was performed on a $3.0 \mathrm{~T}$ GE system using a radio frequency head coil. A T1-weighted image was acquired for landmarking and positioning of subsequent scans. Whole-brain functional scans were acquired using a $\mathrm{T} 2^{*}$-weighted, single shot, reverse-spiral pulse sequence $\left(\mathrm{TR}=2000 \mathrm{~ms}, \mathrm{TE}=30 \mathrm{~ms}\right.$, flip angle $=90^{\circ}, \mathrm{FOV}=22 \mathrm{~cm}$, slice thickness $=3 \mathrm{~mm}, 40$ slices) to minimize susceptibility artifact (Yang et al, 2002). At the beginning of each run, four volumes were discarded to allow for T1 equilibrium.
High-resolution, T1-weighted, inversion-recovery SPGR anatomical images were also collected $(\mathrm{TR}=10.5 \mathrm{~ms}$, $\mathrm{TE}=3.4 \mathrm{~ms}, \quad$ flip angle $=25^{\circ}, \quad \mathrm{FOV}=24 \mathrm{~cm}$, slice thickness $=1.5 \mathrm{~mm}$, number of slices $=106$ ) to facilitate normalization to standard MNI space.

Functional volumes were slice-time corrected, realigned, coregistered within the native anatomy, normalized to standard anatomical space and smoothed using a $5-\mathrm{mm}^{3}$ Gaussian kernel to improve the signal-to-noise ratio. Preprocessing and whole-brain statistical analysis were performed using the statistical parametric mapping (SPM) platform and Matlab.

Functional imaging data were analyzed using a standard SPM hierarchical general linear model approach. On the first level, model parameters were estimated for each conditions within each of the six runs. Contrast images were generated for each subject by applying linear contrasts between combinations of beta estimate images. Contrast images for each emotion were generated using the fixation cross reference (happy $v s$ fixation, sad $v s$ fixation, and neutral $v s$ fixation) and also using neutral emotional stimuli as the reference (happy vs neutral, sad $v s$ neutral). Using both reference, images was adopted as an approach to avoid common inference ambiguities inherent to fMRI imaging in between-groups designs. For example, positive group difference could derive from either differential activation or differential deactivation depending on the contrast. On the group analysis level (second level), contrasts images from the first level were used in emotion-specific SPM full factorial ANOVAs that modeled group (P, SD, ED) and gender (M, F) as factors. Statistical T-test maps were constructed to test for significant activations and deactivations in each group separately (T-test: $\mathrm{P} v s 0, \mathrm{SD} v s 0$, ED $v s$ $0)$. T-test maps were also created to test group differences ( $\mathrm{P} v s \mathrm{SD}, \mathrm{P} v s \mathrm{ED}$ ). Each of these statistical maps was subject to a threshold of $p<0.05$ with family-wise error (FWE) corrections for multiple comparisons and a cluster threshold $(k)$ of 10 voxels. Small volume corrections (SVCs) were also applied to a priori hypothesized regions. For clusters to be considered significant, they were required to meet minimum statistical thresholds of $p<0.001$ uncorrected and a cluster size of 10 voxels and then also meet a FWE-corrected $p$-values of $p<0.05$ within the small volume. Separate second-level multiple regression models were also constructed to test the contribution of salivary cortisol during scanning, valence and arousal ratings to the between-subject variance for first-level contrast images. In these models, average valence and arousal ratings that pertain to the first-level contrasts were used. If the first-level contrast used a neutral control, the differential valence or arousal ratings were used. For example, the difference between the average valence rating of sad stimuli and neutral stimuli were used in the secondlevel model that incorporated the sad $v s$ neutral first level contrast.

Our a priori hypothesis was based on HCT affecting the subgenual cingulate (sgACC), ventral medial prefrontal cortex (VMPFC), and amygdala. As this hypothesis was specific to anatomical regions, we also compared groups using a region of interest (ROI) approach. In the ROI approach, functional activation from first-level contrasts is averaged across the anatomical space. ROI masks were 
derived from the MNI Space Utility (http://www.ihb.spb. $\mathrm{ru} / \sim$ pet_lab/MSU/MSUMain.html) and Anatomical Automatic Labeling software (Tzourio-Mazoyer et al, 2002). ROI spatial definitions were verified using three separate published brain atlases (Damasio, 2005; Mai et al, 2004; Sudheimer et al., 2002). The ROIs used were the bilateral rectal gyrus (VMPFC), Brodmann area 25 (sgACC), and amygdala. Contrast value averages were extracted using modified versions of SPM5/8 code and the Neurotools extension (Imfeld, 2009). Contrast extractions were performed for each ROI for each subject. These values were analyzed in contrast-specific multivariate ANOVAs using SPSS. Each of these models included ROI extractions from each brain region (sgACC, VMPFC, amygdala). They also included group (P, SD, ED) and gender as between group factors and used subject age and weight as covariates. Post-hoc comparisons were subject to Bonferroni corrections.

\section{Subjective Awareness of HCT Administration}

At the completion of each subject's involvement in the study, they were asked to guess if they had been assigned to the P, SD, or ED group. Each subject's guess was recorded, and response patterns from each group was analyzed using a Chi-square analysis.

\section{RESULTS}

\section{HCT Administration and Brain Activation}

Sadness-related brain activation was decreased in sgACC and VMPFC in one or both of the HCT groups compared with the P group. These group differences were not detected on whole-brain FWE-corrected maps but were apparent at FWE $(p<0.05)$ after SVCs and also in ROI contrast extractions (Figure 1, Table 1, Table 2). HCT did not affect amygdala during sadness. Happiness related activation was increased in the sgACC and the amygdala for the SD group. This increase was apparent for happy faces, but not happy IAPS. HCT administration did not have any effects on brain activation evoked by neutral stimuli in any brain region. For both the happy and neutral conditions, no voxels survived FWE corrections for any group-level contrasts. The significant findings for happy faces were only apparent in the ROI contrast extractions.

\section{Subgenual Cingulate}

When comparing sgACC activations from the groups using the sad $v$ s fixation contrast, the $\mathrm{P}$ group demonstrated activation of the sgACC in response to viewing sad stimuli. The HCT groups, however, showed deactivation of the sgACC to sad stimuli (Figure 1, Figure 2). There was a significant omnibus effect of group on sgACC activation, which was driven by decreased activation in both the SD group and the ED group relative to the $\mathrm{P}$ group (Table 1). These effects were significant after SVC at FWE rate of $p<0.05$ (Table 2) and in the ROI extractions (Figure 1, Table 1). Furthermore, the multiple regression model showed a negative relationship between average cortisol measured at scan time and sgACC activity across subjects (Table 2).
When comparing sgACC activations from the groups using the sad $v s$ neutral contrast similar results were observed. The $\mathrm{P}$ group showed activation, the SD group showed no response, and the ED group showed a deactivation. There was a significant omnibus effect of group, which was driven by significantly decreased activation in the ED group but not the SD group. (Table 1). These effects were significant after SVC at FWE rate of $p<0.05$ (Table 2) and in the ROI extractions (Figure 1, Table 1), but not in the multiple regression model.

The ROI analysis also detected an increase in sgACC activation in the SD group relative to the $\mathrm{P}$ group when comparing activation induced by happy faces relative to neutral faces. This effect was significant in the ROI analysis as a Bonferroni-corrected post-hoc t-test, but not after a SVC, and not in the multiple regression model. This effect is the result of an interaction between a non-significant increase in the sgACC response to the happy faces and a decrease in the sgACC response to neutral faces (Table 1 and Figure 1 row 1, column 2).

\section{Ventral Medial Prefrontal Cortex}

When comparing VMPFC activations from the groups using the sad $v$ s fixation contrast, the $\mathrm{P}$ group demonstrated activation of the VMPFC, the SD group showed no response, and the ED showed a deactivation (Figure 1). There was a significant omnibus effect of group on VMPFC activation, which was driven by decreased activation in the SD group and the ED group relative to the $\mathrm{P}$ group (Figure 1, Table 1)). These effects were significant after SVC at FWE rate of $p<0.05$ (Table 2), in the ROI extractions (Table 1 ), and in the multiple regression model.

When comparing VMPFC activations from the groups using the sad $v s$ neutral contrast the results were similar although less robust than the sad $v s$ fixation contrast. The $\mathrm{P}$ group demonstrated an activation of the VMPFC, the SD group showed no response, and the $\mathrm{ED}$ showed a deactivation. The omnibus effect of group was not significant. The SD group did not show significantly less activation than the P group, but the ED group did (Table 1). These results were significant after SVC at FWE rate of $p<0.05$ (Table 2), in the ROI extractions (Table 1), but not in the multiple regression model.

\section{Amygdala}

No significant group effects were observed in the amygdala using either the sad $v s$ fixation or the sad $v s$ neutral contrasts. A qualitative stepwise decrease across the groups was seen in the sad $v s$ neutral contrast with the P group showing the strongest activation, the SD showing less activation, and the ED group showing the least activation (Figure 1). When comparing amygdala activations from the groups on the happy faces $v s$ neutral faces contrast, there was a significant omnibus effect of group, driven by increased activation in the SD group. However, this difference did not survive Bonferroni correction (Table 1). This effect is the result of an interaction between a nonsignificant increase in the amygdala response to the happy faces and a decrease in the amygdala response to neutral faces (Figure 1 row 3, column 2). 
Table I Group Differences are Shown for Emotion Evoked Brain Activations in Our Regions of Interest Hydrocortisone effect on contrasts using both faces and IAPS Hydrocortisone effect on contrasts using faces only pictures

\begin{tabular}{|c|c|c|c|c|c|c|c|c|c|}
\hline Contrast & Region & Test & $F / T$ value & $p$-value & Contrast & Region & Test & $F / T$ value & $p$-value \\
\hline \multicolumn{10}{|l|}{ Sad stimuli } \\
\hline \multirow[t]{11}{*}{$\begin{array}{l}\text { All sad stimuli } \\
\text { fixation }\end{array}$} & & & & & $\begin{array}{l}\text { Sad faces } \\
\text { vs fixation }\end{array}$ & & & & \\
\hline & Multivariate & Pillai's & $F(6,102)=1.92$ & 0.0851 & & Multivariate & Pillai's & $F(6,102)=0.42$ & 0.8623 \\
\hline & sgACC & Omnibus & $F(2,52)=4.32$ & $0.0184 *$ & & sgACC & Omnibus & $F(2,52)=0.83$ & 0.4425 \\
\hline & & $\begin{array}{l}\text { Single dose } \\
\text { vs placebo }\end{array}$ & $T(40)=-2.55$ & $0.0274 *$ & & & $\begin{array}{l}\text { Single dose } \\
\text { vs placebo }\end{array}$ & $T(40)=-1.06$ & 0.5838 \\
\hline & & $\begin{array}{l}\text { Extended } \\
\text { dose vs } \\
\text { placebo }\end{array}$ & $T(38)=-2.53$ & $0.0286 *$ & & & $\begin{array}{l}\text { Extended } \\
\text { dose vs } \\
\text { placebo }\end{array}$ & $T(38)=-1.16$ & 0.5046 \\
\hline & VMPFC & Omnibus & $F(2,52)=5.46$ & 0.007 I* & & VMPFC & Omnibus & $F(2,52)=0.84$ & 0.4355 \\
\hline & & $\begin{array}{l}\text { Single dose } \\
\text { vs placebo }\end{array}$ & $T(40)=-2.55$ & $0.0274^{*}$ & & & $\begin{array}{l}\text { Single dose } \\
\text { vs placebo }\end{array}$ & $T(40)=-0.80$ & 0.8596 \\
\hline & & $\begin{array}{l}\text { Extended } \\
\text { dose vs } \\
\text { placebo }\end{array}$ & $T(38)=-3.09$ & $0.0064 *$ & & & $\begin{array}{l}\text { Extended } \\
\text { dose vs } \\
\text { placebo }\end{array}$ & $T(38)=-1.29$ & 0.4072 \\
\hline & Amygdala & Omnibus & $F(2,52)=1.25$ & 0.2958 & & Amygdala & Omnibus & $F(2,52)=0.04$ & 0.9619 \\
\hline & & $\begin{array}{l}\text { Single dose } \\
\text { vs placebo }\end{array}$ & $T(40)=-1.53$ & 0.2652 & & & $\begin{array}{l}\text { Single dose } \\
\text { vs placebo }\end{array}$ & $T(40)=-0.27$ & 1.0000 \\
\hline & & $\begin{array}{l}\text { Extended } \\
\text { dose vs } \\
\text { placebo }\end{array}$ & $T(38)=-1.11$ & 0.5458 & & & $\begin{array}{l}\text { Extended } \\
\text { dose vs } \\
\text { placebo }\end{array}$ & $T(38)=-0.18$ & 1.0000 \\
\hline \multirow[t]{10}{*}{$\begin{array}{l}\text { All sad stimuli v } \\
\text { all neutral stimu }\end{array}$} & & & & & $\begin{array}{l}\text { Sad faces } \\
\text { vs neutral faces }\end{array}$ & & & & \\
\hline & Multivariate & Pillai's & $F(6,102)=1.13$ & & & Multivariate & Pillai's & $F(6,102)=0.63$ & \\
\hline & sgACC & Omnibus & $F(2,52)=3.19$ & $0.0492 *$ & & sgACC & Omnibus & $F(2,52)=1.52$ & 0.2284 \\
\hline & & $\begin{array}{l}\text { Single dose } \\
\text { vs placebo }\end{array}$ & $T(40)=-1.00$ & 0.6414 & & & $\begin{array}{l}\text { Single dose } \\
\text { vs placebo }\end{array}$ & $T(40)=0.50$ & 1.0000 \\
\hline & & $\begin{array}{l}\text { Extended } \\
\text { dose vs } \\
\text { placebo }\end{array}$ & $T(38)=-2.51$ & $0.0304 *$ & & & $\begin{array}{l}\text { Extended } \\
\text { dose vs } \\
\text { placebo }\end{array}$ & $T(38)=-1.20$ & 0.4748 \\
\hline & VMPFC & Omnibus & $F(2,52)=2.76$ & 0.0726 & & VMPFC & Omnibus & $F(2,52)=0.90$ & 0.4124 \\
\hline & & $\begin{array}{l}\text { Single dose } \\
\text { vs placebo }\end{array}$ & $T(40)=-1.07$ & 0.5784 & & & $\begin{array}{l}\text { Single dose } \\
\text { vs placebo }\end{array}$ & $T(40)=0.54$ & 1.0000 \\
\hline & & $\begin{array}{l}\text { Extended } \\
\text { dose vs } \\
\text { placebo }\end{array}$ & $T(38)=-2.35$ & $0.0456 *$ & & & $\begin{array}{l}\text { Extended } \\
\text { dose vs } \\
\text { placebo }\end{array}$ & $T(38)=-0.80$ & 0.8550 \\
\hline & Amygdala & Omnibus & $F(2,52)=0.94$ & 0.3967 & & Amygdala & Omnibus & $F(2,52)=0.98$ & 0.3810 \\
\hline & & $\begin{array}{l}\text { Single dose } \\
\text { vs placebo }\end{array}$ & $T(40)=-0.03$ & 1.0000 & & & $\begin{array}{l}\text { Single dose } \\
\text { vs placebo }\end{array}$ & $T(40)=1.02$ & 0.6270 \\
\hline
\end{tabular}

Hydrocortisone effect on contrasts using IAPS pictures only

Contrast Region Test $\quad F / T$ value $\quad p$-value

Sad IAPS

vs fixation

Multivariate Pillai's $\quad F(6,102)=1.29 \quad 0.2696$

sgACC Omnibus $F(2,52)=3.56 \quad \mathbf{0 . 0 3 5 5 *}$

Single dose $T(40)=-2.36 \quad \mathbf{0 . 0 4 4 2} *$

us placebo

0.0564

Extended $\quad T(38)=-2.26 \quad 0.0564$

dose vs

placebo

VMPFC Omnibus $F(2,52)=3.47 \quad \mathbf{0 . 0 3 8 6 *}$

Single dose $T(40)=-2.16 \quad 0.0708$

vs placebo

Extended $T(38)=-2.38 \quad \mathbf{0 . 0 4 1 \mathbf { 8 } ^ { * }}$

dose vs

placebo

Amygdala

Omnibus

Single dose $T(40)=-1.46 \quad 0.2984$

Extended $\quad T(38)=-1.08 \quad 0.5692$

dose vs

placebo

Sad IAPS vs

neutral IAPS

$\begin{array}{llll}\text { Multivariate } & \text { Pillai's } & F(6,102)=0.58 & \\ \text { sgACC } & \text { Omnibus } & F(2,52)=1.82 & 0.1715 \\ & \begin{array}{l}\text { Single dose } \\ \text { vs placebo }\end{array} & T(40)=-1.56 & 0.2510 \\ & \begin{array}{l}\text { Extended } \\ \text { dose vs }\end{array} & T(38)=-1.74 & 0.1770 \\ \text { placebo } & & \\ \text { VMPFC } & \text { Omnibus } & F(2,52)=1.53 & 0.2259 \\ & \begin{array}{l}\text { Single dose } \\ \text { vs placebo }\end{array} & T(40)=-1.47 & 0.2960 \\ & \begin{array}{l}\text { Extended } \\ \text { dose vs }\end{array} & T(38)=-1.56 & 0.2510 \\ & \text { placebo } & & \\ \text { Amygdala } & \text { Omnibus } & F(2,52)=0.73 & 0.4857 \\ & \text { Single dose } & T(40)=-1.02 & 0.6286 \\ & \text { vs placebo } & & \end{array}$

vs placebo 
Table I (Continued)

Hydrocortisone effect on contrasts using both faces and IAPS

Hydrocortisone effect on contrasts using faces only

Hydrocortisone effect on contrasts using IAPS pictures only pictures

\begin{tabular}{lllll}
\hline Contrast & Region & Test & F/T value & p-value \\
\hline & $\begin{array}{l}\text { Extended } \\
\text { dose vs } \\
\text { placebo }\end{array}$ & $T(38)=-1.20$ & 0.4696 \\
& & & \\
& &
\end{tabular}

Happy stimuli

All happy stimuli

vs fixation

\begin{tabular}{l} 
Contrast \\
\hline \\
Happy faces \\
vs fixation
\end{tabular}

Region

\begin{tabular}{lll} 
Test & $\boldsymbol{F} / \boldsymbol{T}$ value & $\boldsymbol{p}$-value \\
\hline $\begin{array}{l}\text { Extended } \\
\text { dose vs } \\
\text { placebo }\end{array}$ & $T(38)=-0.33$ & 1.0000 \\
& & \\
\hline
\end{tabular}

\begin{tabular}{|c|c|c|c|c|}
\hline Contrast & Region & Test & $F / T$ value & $p$-value \\
\hline & & $\begin{array}{l}\text { Extended } \\
\text { dose vs } \\
\text { placebo }\end{array}$ & $T(38)=-1.08$ & 0.5732 \\
\hline \multicolumn{5}{|c|}{$\begin{array}{l}\text { Happy IAPS } \\
\text { vs fixation }\end{array}$} \\
\hline & Multivariate & Pillai's & $F(6,102)=0.59$ & \\
\hline & sgACC & Omnibus & $F(2,52)=0.84$ & 0.4367 \\
\hline & & $\begin{array}{l}\text { Single dose } \\
\text { vs placebo }\end{array}$ & $T(40)=-0.87$ & 0.7806 \\
\hline & & $\begin{array}{l}\text { Extended } \\
\text { dose vs } \\
\text { placebo }\end{array}$ & $T(38)=-1.27$ & 0.4200 \\
\hline & VMPFC & Omnibus & $F(2,52)=1.43$ & 0.2481 \\
\hline & & $\begin{array}{l}\text { Single dose } \\
\text { vs placebo }\end{array}$ & $T(40)=-1.37$ & 0.3534 \\
\hline & & $\begin{array}{l}\text { Extended } \\
\text { dose vs } \\
\text { placebo }\end{array}$ & $T(38)=-1.54$ & 0.2570 \\
\hline & Amygdala & Omnibus & $F(2,52)=0.33$ & 0.7197 \\
\hline & & $\begin{array}{l}\text { Single dose } \\
\text { vs placebo }\end{array}$ & $T(40)=-0.67$ & 1.0000 \\
\hline & & $\begin{array}{l}\text { Extended } \\
\text { dose vs } \\
\text { placebo }\end{array}$ & $T(38)=-0.73$ & 0.9342 \\
\hline \multicolumn{5}{|c|}{$\begin{array}{l}\text { Happy IAPS } \\
\text { vs neutral IAPS }\end{array}$} \\
\hline & Multivariate & Pillai's & $F(6,102)=0.53$ & \\
\hline & $\operatorname{sgACC}$ & Omnibus & $F(2,52)=0.46$ & 0.6311 \\
\hline & & $\begin{array}{l}\text { Single dose } \\
\text { vs placebo }\end{array}$ & $T(40)=-0.35$ & 1.0000 \\
\hline & & $\begin{array}{l}\text { Extended } \\
\text { dose vs } \\
\text { placebo }\end{array}$ & $T(38)=-0.95$ & 0.6906 \\
\hline & VMPFC & Omnibus & $F(2,52)=0.66$ & 0.5233 \\
\hline & & $\begin{array}{l}\text { Single dose } \\
\text { vs placebo }\end{array}$ & $T(40)=-0.96$ & 0.6876 \\
\hline & & $\begin{array}{l}\text { Extended } \\
\text { dose vs } \\
\text { placebo }\end{array}$ & $T(38)=-1.02$ & 0.6214 \\
\hline
\end{tabular}

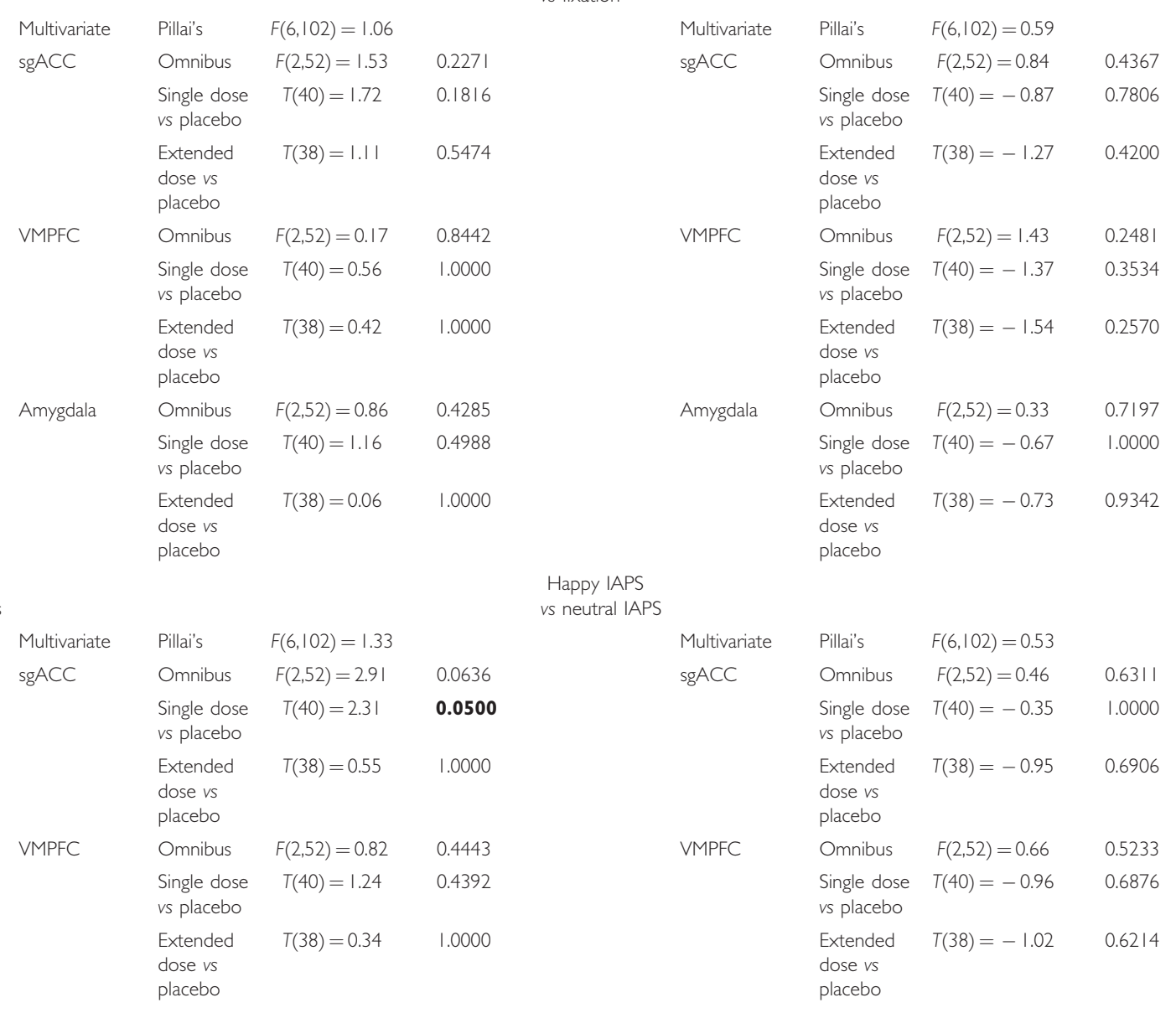

$\begin{array}{llll}\text { Multivariate } & \text { Pillai's } & F(6,102)=0.59 & \\ \text { sgACC } & \begin{array}{l}\text { Omnibus } \\ \text { Single dose }\end{array} & F(2,52)=0.24 & 0.7848 \\ & \begin{array}{l}\text { vs placebo } \\ \text { Extended } \\ \text { dose vs } \\ \text { placebo }\end{array} & T(38)=-43 & 1.0000 \\ \text { VMPFC } & \begin{array}{l}\text { Omnibus } \\ \text { Single dose }\end{array} & F(2,52)=0.63 & 1.0000 \\ & \begin{array}{l}\text { vs placebo } \\ \text { Amygdala }\end{array} & 0.5392 \\ & \begin{array}{l}\text { Extended } \\ \text { dose vs }\end{array} & T(38)=-1.07 & 0.8384 \\ & \begin{array}{l}\text { Placebo } \\ \text { Omnibus }\end{array} & F(2,52)=0.40 & 0.5790 \\ & \begin{array}{l}\text { Single dose } \\ \text { vs placebo }\end{array} & T(40)=0.47 & 1.0748 \\ & \begin{array}{l}\text { Extended } \\ \text { dose vs }\end{array} & T(38)=-0.42 & 1.0000 \\ \text { placebo } & & \\ & & & \end{array}$

$\begin{array}{llll}\text { Multivariate } & \text { Pillai's } & F(6,102)=1.16 & \\ \text { sgACC } & \text { Omnibus } & F(2,52)=2.52 & 0.0899 \\ & \begin{array}{l}\text { Single dose } \\ \text { vs placebo }\end{array} & T(40)=1.77 & 0.1664 \\ & \begin{array}{l}\text { Extended } \\ \text { dose vs }\end{array} & T(38)=-0.32 & 1.0000 \\ & \text { placebo } & & \\ \text { VMPFC } & \begin{array}{l}\text { Omnibus } \\ \text { Single dose }\end{array} & F(2,52)=0.54 & 0.5841 \\ & \begin{array}{l}\text { vs placebo } \\ \text { Extended }\end{array} & T(38)=0.29 & 1.0000 \\ \text { dose vs } & & \\ \text { placebo } & & 0.9424 \\ & & & \end{array}$

\section{Happy faces} vs neutral faces

Happy vs neutra 
Table I (Continued)

\begin{tabular}{|c|c|c|c|c|c|c|c|c|c|c|c|c|c|c|}
\hline \multicolumn{5}{|c|}{$\begin{array}{l}\text { Hydrocortisone effect on contrasts using both faces and IAPS } \\
\text { pictures }\end{array}$} & \multicolumn{5}{|c|}{ Hydrocortisone effect on contrasts using faces only } & \multicolumn{5}{|c|}{ Hydrocortisone effect on contrasts using IAPS pictures only } \\
\hline Contrast & Region & Test & $F / T$ value & $p$-value & Contrast & Region & Test & $F / T$ value & $p$-value & Contrast & Region & Test & $F / T$ value & $p$-value \\
\hline & Amygdala & Omnibus & $F(2,52)=2.20$ & 0.1210 & & Amygdala & Omnibus & $F(2,52)=3.26$ & $0.0464 *$ & & Amygdala & Omnibus & $F(2,52)=0.58$ & 0.5651 \\
\hline & & $\begin{array}{l}\text { Single dose } \\
\text { vs placebo }\end{array}$ & $T(40)=1.31$ & 0.3888 & & & $\begin{array}{l}\text { Single dose } \\
\text { vs placebo }\end{array}$ & $T(40)=2.12$ & 0.0768 & & & $\begin{array}{l}\text { Single dose } \\
\text { vs placebo }\end{array}$ & $T(40)=-0.57$ & 1.0000 \\
\hline & & $\begin{array}{l}\text { Extended } \\
\text { dose vs } \\
\text { placebo }\end{array}$ & $T(38)=-0.76$ & 0.8998 & & & $\begin{array}{l}\text { Extended } \\
\text { dose vs } \\
\text { placebo }\end{array}$ & $T(38)=-0.17$ & 1.0000 & & & $\begin{array}{l}\text { Extended } \\
\text { dose vs } \\
\text { placebo }\end{array}$ & $T(38)=-1.07$ & 0.5760 \\
\hline \multicolumn{15}{|c|}{ Neutral stimuli } \\
\hline \multirow[t]{11}{*}{$\begin{array}{l}\text { Neutral vs } \\
\text { fixation }\end{array}$} & & & & & $\begin{array}{l}\text { Neutral faces } \\
\text { vs fixation }\end{array}$ & & & & & $\begin{array}{l}\text { Neutral IAPS vs } \\
\text { fixation }\end{array}$ & & & & \\
\hline & Multivariate & Pillai's & $F(6,102)=1.04$ & & & Multivariate & Pillai's & $F(6,102)=0.74$ & & & Multivariate & Pillai's & $F(6,102)=0.21$ & \\
\hline & sgACC & Omnibus & $F(2,52)=1.82$ & 0.1729 & & sgACC & Omnibus & $F(2,52)=1.55$ & 0.2226 & & sgACC & Omnibus & $F(2,52)=0.20$ & 0.8223 \\
\hline & & $\begin{array}{l}\text { Single dose } \\
\text { vs placebo }\end{array}$ & $T(40)=-1.61$ & 0.2258 & & & $\begin{array}{l}\text { Single dose } \\
\text { vs placebo }\end{array}$ & $T(40)=-1.38$ & 0.3464 & & & $\begin{array}{l}\text { Single dose } \\
\text { vs placebo }\end{array}$ & $T(40)=-0.62$ & 1.0000 \\
\hline & & $\begin{array}{l}\text { Extended } \\
\text { dose vs } \\
\text { placebo }\end{array}$ & $T(38)=0.08$ & 1.0000 & & & $\begin{array}{l}\text { Extended } \\
\text { dose vs } \\
\text { placebo }\end{array}$ & $T(38)=0.26$ & 1.0000 & & & $\begin{array}{l}\text { Extended } \\
\text { dose vs } \\
\text { placebo }\end{array}$ & $T(38)=-0.22$ & 1.0000 \\
\hline & VMPFC & Omnibus & $F(2,52)=0.87$ & 0.4263 & & VMPFC & Omnibus & $F(2,52)=0.80$ & 0.4567 & & VMPFC & Omnibus & $F(2,52)=0.10$ & 0.9031 \\
\hline & & $\begin{array}{l}\text { Single dose } \\
\text { vs placebo }\end{array}$ & $T(40)=-1.29$ & 0.4068 & & & $\begin{array}{l}\text { Single dose } \\
\text { vs placebo }\end{array}$ & $T(40)=-1.12$ & 0.5324 & & & $\begin{array}{l}\text { Single dose } \\
\text { vs placebo }\end{array}$ & $T(40)=-0.31$ & 1.0000 \\
\hline & & $\begin{array}{l}\text { Extended } \\
\text { dose vs } \\
\text { placebo }\end{array}$ & $T(38)=-0.41$ & 1.0000 & & & $\begin{array}{l}\text { Extended } \\
\text { dose vs } \\
\text { placebo }\end{array}$ & $T(38)=-0.06$ & 1.0000 & & & $\begin{array}{l}\text { Extended } \\
\text { dose vs } \\
\text { placebo }\end{array}$ & $T(38)=-0.44$ & 1.0000 \\
\hline & Amygdala & Omnibus & $F(2,52)=1.49$ & 0.2358 & & Amygdala & Omnibus & $F(2,52)=1.89$ & 0.1614 & & Amygdala & Omnibus & $F(2,52)=0.14$ & 0.8678 \\
\hline & & $\begin{array}{l}\text { Single dose } \\
\text { vs placebo }\end{array}$ & $T(40)=-1.16$ & 0.4990 & & & $\begin{array}{l}\text { Single dose } \\
\text { vs placebo }\end{array}$ & $T(40)=-1.53$ & 0.2636 & & & $\begin{array}{l}\text { Single dose } \\
\text { vs placebo }\end{array}$ & $T(40)=-0.02$ & 1.0000 \\
\hline & & $\begin{array}{l}\text { Extended } \\
\text { dose vs } \\
\text { placebo }\end{array}$ & $T(38)=0.52$ & 1.0000 & & & $\begin{array}{l}\text { Extended } \\
\text { dose vs } \\
\text { placebo }\end{array}$ & $T(38)=0.27$ & 1.0000 & & & $\begin{array}{l}\text { Extended } \\
\text { dose vs } \\
\text { placebo }\end{array}$ & $T(38)=0.45$ & 1.0000 \\
\hline
\end{tabular}

Multivariate (Pillai's) tests detect group differences across all three brain regions included in the analysis (Subgenual cingulate sgACC, Ventral medial prefrontal cortex VMPFC, and the amygdala). Omnibus effects of group (F-tests) and individual group differences ( $T$-tests) are included.

$T$-test values indicate the direction of group differences.

Positive significant $T$ values indicate the placebo group had higher evoked response than the single dose or extended dose group.

Negative $T$ values indicate a higher evoked response in the placebo group.

Listed p-values are Bonferroni adjusted for multiple comparisons.

*Bold value indicate $p<0.05$ for omnibus tests or after Bonferroni correction. 
Table 2 Whole-Brain Results Demonstrating Group Differences in Brain Activation Evoked by Emotion Conditions

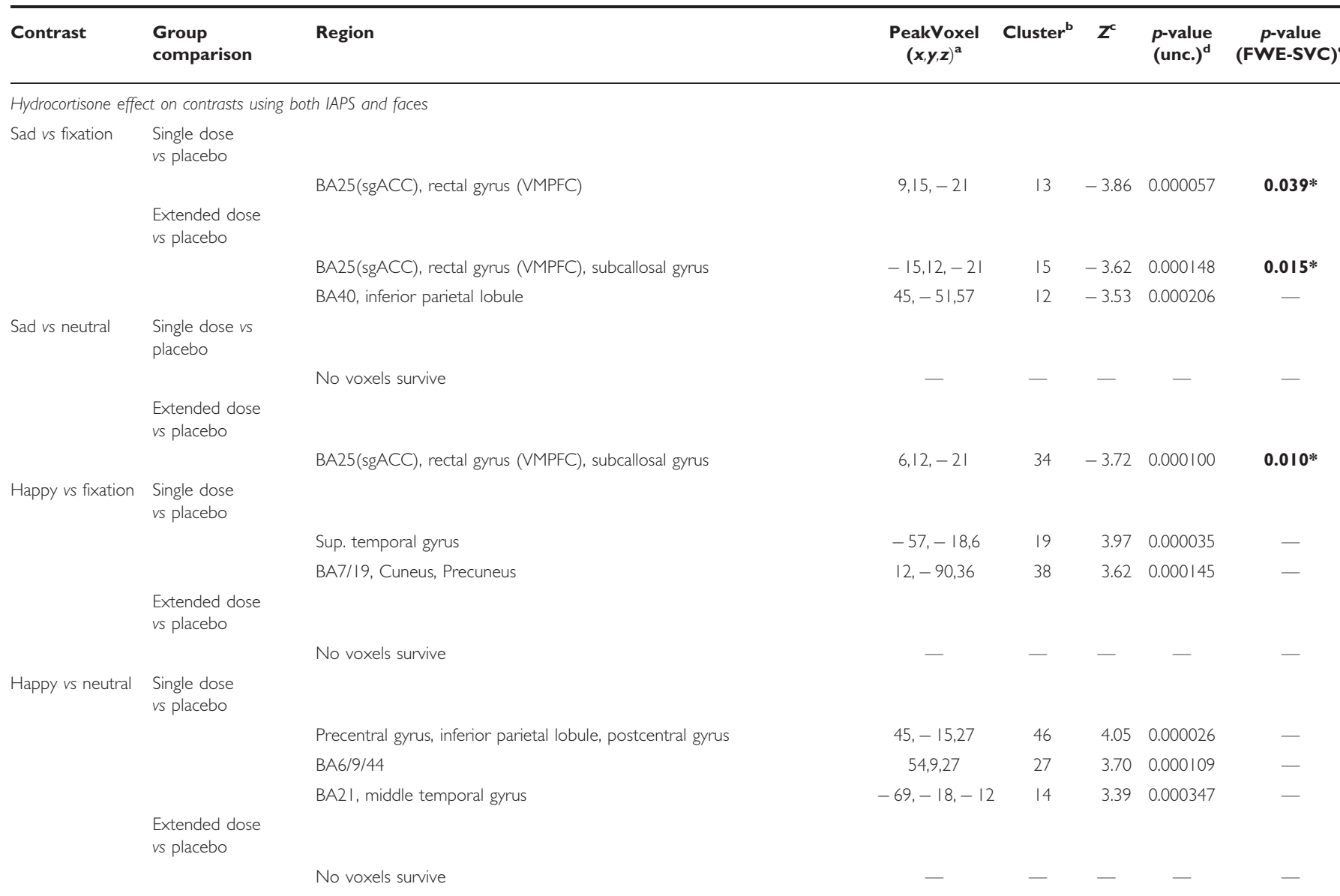

Hydrocortisone effect on contrasts using faces only

Sad faces vs Single dose

fixation vs placebo

BA40, inferior parietal lobule

BA40, inferior parietal lobule

BA $8 / 19$, middle occipital gyrus

Extended dose

vs placebo

Sad faces vs Single dose

neutral faces vs placebo

Extended dose

vs placebo

Happy faces vs Single dose

fixation vs placebo

BA40, inferior parietal lobule

BA40, inferior parietal lobule

BA40, inferior parietal lobule

$\mathrm{BA} \mid 7$, lingual gyrus
$\mathrm{BA} 19$, cuneus, precuneus

Sub - gyral,temporal lobe white matter

BA22, middle temporal gyrus, superior temporal gyrus

BAI7/19, superior occipital gyrus, cuneus, precuneus

BA20, fusiform gyrus, inferior temporal gyrus

BA28/34

BA40/4I, superior temporal gyrus

BA39, middle temporal gyrus, superior temporal gyrus

Extended dose

vs placebo

\begin{tabular}{|c|c|c|c|}
\hline$-54,-60,45$ & 18 & -3.88 & 0.000053 \\
\hline$-57,-57,45$ & 17 & -3.74 & 0.000092 \\
\hline$-24,-102,6$ & 11 & -3.56 & 0.000186 \\
\hline $48,-51,57$ & 10 & -3.54 & 0.000201 \\
\hline $54,-36,30$ & 10 & 3.80 & $0.00007 \mid$ \\
\hline $57,-5|, 5|$ & 10 & -3.76 & 0.000086 \\
\hline $0,-96,-6$ & 15 & -3.62 & 0.000149 \\
\hline $15,-90,42$ & 37 & 4.07 & 0.000024 \\
\hline$-33,-54,-3$ & 18 & 4.05 & 0.000025 \\
\hline$-60,-15,3$ & 25 & 3.98 & 0.000034 \\
\hline$-15,-84,42$ & 89 & 3.95 & 0.000039 \\
\hline $51,-27,-27$ & 15 & 3.94 & 0.000041 \\
\hline$-15,0,-27$ & 15 & 3.87 & 0.000055 \\
\hline $54,-24,12$ & 26 & 3.78 & 0.000080 \\
\hline $45,-63,15$ & 10 & 3.52 & 0.000218 \\
\hline
\end{tabular}


Table 2 (Continued)

\begin{tabular}{|c|c|c|c|c|c|c|c|}
\hline Contrast & $\begin{array}{l}\text { Group } \\
\text { comparison }\end{array}$ & Region & $\begin{array}{l}\text { PeakVoxel } \\
(x, y, z)^{a}\end{array}$ & Cluster $^{\mathrm{b}}$ & $z^{c}$ & $\begin{array}{l}p \text {-value } \\
\text { (unc.) }^{\text {d }}\end{array}$ & $\begin{array}{c}p \text {-value } \\
\text { (FWE-SVC }\end{array}$ \\
\hline \multirow[t]{15}{*}{$\begin{array}{l}\text { Happy faces vs } \\
\text { neutral faces }\end{array}$} & $\begin{array}{l}\text { Single dose } \\
\text { vs placebo }\end{array}$ & & & & & & \\
\hline & & BA2/3/4/6/40/44, inferior parietal lobule, postcentral gyrus, precentral gyrus & $48,-18,27$ & 211 & 4.94 & 0.0000004 & - \\
\hline & & $\begin{array}{l}\text { BA13/20/22/36/37, inferior temporal gyrus, parahippocampal gyrus, middle } \\
\text { temporal gyrus, superior temporal gyrus }\end{array}$ & $-45,-30,-21$ & 171 & 4.24 & 0.000011 & - \\
\hline & & BA20, inferior temporal gyrus, middle temporal gyrus & $51,-27,-24$ & 43 & 4.20 & 0.000014 & - \\
\hline & & BA22, middle temporal gyrus, superior temporal gyrus & $-60,-15,0$ & 22 & 3.88 & 0.000051 & - \\
\hline & & BAI9, middle occipital gyrus & $51,-84,0$ & 13 & 3.88 & 0.000052 & - \\
\hline & & BA37, inferior temporal gyrus & $45,-39,-24$ & 13 & 3.88 & 0.000052 & - \\
\hline & & Cerebellar culmen & $21,-39,-30$ & 11 & 3.82 & 0.000067 & - \\
\hline & & BA21, middle temporal gyrus, superior temporal gyrus & $66,-6,-3$ & 11 & 3.63 & 0.000144 & - \\
\hline & & BA44, inferior frontal gyrus & $-51,12,12$ & 13 & 3.62 & $0.000 \mid 48$ & - \\
\hline & & BA20 & $-33,-9,-39$ & 11 & 3.57 & 0.000176 & - \\
\hline & & BA6/9, inferior frontal gyrus & $-57,-3,39$ & 28 & 3.51 & 0.000222 & - \\
\hline & & BAI3/4I, insula, superior temporal gyrus & $-42,-12,3$ & 23 & 3.42 & 0.000316 & - \\
\hline & $\begin{array}{l}\text { Extended dose } \\
\text { vs placebo }\end{array}$ & & & & & & \\
\hline & & Fusiform gyrus & $-42,-12,-21$ & 13 & 3.68 & 0.000119 & - \\
\hline \multicolumn{8}{|c|}{ Hydrocortisone effect on contrast using IAPS only } \\
\hline \multirow[t]{5}{*}{$\begin{array}{l}\text { Sad IAPS vs } \\
\text { fixation }\end{array}$} & $\begin{array}{l}\text { Single dose } \\
\text { vs placebo }\end{array}$ & & & & & & \\
\hline & & No voxels survive & - & - & - & - & - \\
\hline & $\begin{array}{l}\text { Extended dose } \\
\text { vs placebo }\end{array}$ & & & & & & \\
\hline & & BA25(sgACC), subcallosal gyrus, rectal gyrus (VMPFC) & $-15,12,-21$ & 13 & -3.59 & 0.000162 & $0.016 *$ \\
\hline & & BA40, inferior parietal lobule & $39,-51,51$ & 16 & -3.53 & 0.000204 & \\
\hline \multirow[t]{4}{*}{$\begin{array}{l}\text { Sad IAPS vs } \\
\text { neutral IAPS }\end{array}$} & $\begin{array}{l}\text { Single dose } \\
\text { vs placebo }\end{array}$ & & & & & & \\
\hline & & No voxels survive & - & - & - & - & - \\
\hline & $\begin{array}{l}\text { Extended dose } \\
\text { vs placebo }\end{array}$ & & & & & & \\
\hline & & No voxels survive & - & - & - & - & - \\
\hline \multirow[t]{5}{*}{$\begin{array}{l}\text { Happy IAPS vs } \\
\text { fixation }\end{array}$} & $\begin{array}{l}\text { Single dose } \\
\text { vs placebo }\end{array}$ & & & & & & \\
\hline & & No voxels survive & - & - & - & - & - \\
\hline & $\begin{array}{l}\text { Extended dose } \\
\text { vs placebo }\end{array}$ & & & & & & \\
\hline & & BAI8/19, fusiform gyrus & $-27,-72,-18$ & 23 & -3.72 & 0.000100 & - \\
\hline & & BAI8, cuneus & $-12,-93,6$ & 21 & -3.62 & $0.000 \mid 47$ & \\
\hline \multirow[t]{10}{*}{$\begin{array}{l}\text { Happy IAPS vs } \\
\text { neutral IAPS }\end{array}$} & $\begin{array}{l}\text { Single dose } \\
\text { vs placebo }\end{array}$ & & & & & & \\
\hline & & BA $18 / 19$, middle occipital gyrus & $42,-78,-15$ & 10 & -3.69 & 0.000114 & - \\
\hline & $\begin{array}{l}\text { Extended dose } \\
\text { vs placebo }\end{array}$ & & & & & & \\
\hline & & BAI8/I8/37, middle occipital gyrus, lingual gyrus, fusiform gyrus & $-33,-60,-18$ & 58 & -3.83 & 0.000065 & - \\
\hline & & BAI $8 / 18 / 37$, inferior occipital gyrus, lingual gyrus, fusiform gyrus & $30,-84,-15$ & 26 & -3.72 & 0.000101 & - \\
\hline & & BA $18 / \mid 9$, middle occipital gyrus & $45,-93,0$ & 30 & -3.69 & 0.000113 & - \\
\hline & & BAI9, cuneus & $-24,-90,9$ & 10 & -3.55 & 0.000194 & - \\
\hline & & BAI8/19, inferior occipital gyrus, middle occipital gyrus & $39,-72,-9$ & 19 & -3.48 & 0.000249 & - \\
\hline & & BA22, middle temporal gyrus & $36,-60,15$ & 13 & -3.39 & 0.000346 & - \\
\hline & & BAI8, middle occipital gyrus, cuneus & $-12,-102,15$ & 16 & -3.34 & 0.000426 & - \\
\hline
\end{tabular}

Multiple regression on contrasts using both IAPS and faces

Sad vs fixation Cortisol

on contrast
ingle dos
s placebo
Etended
s placebo
ingle dos
Ex placebo
ingle dos
s placebo
Ex

Negative effect of BA25(sgACC), rectal gyrus (VMPFC), subcallosal gyrus

$6,12,-18 \quad 22 \quad-4.27 \quad 0.000010$

$0.001 *$

Positive effect of Cerebellum

$21,-93,-30 \quad 18 \quad 3.83 \quad 0.000065$

cortisol 
Table 2 (Continued)

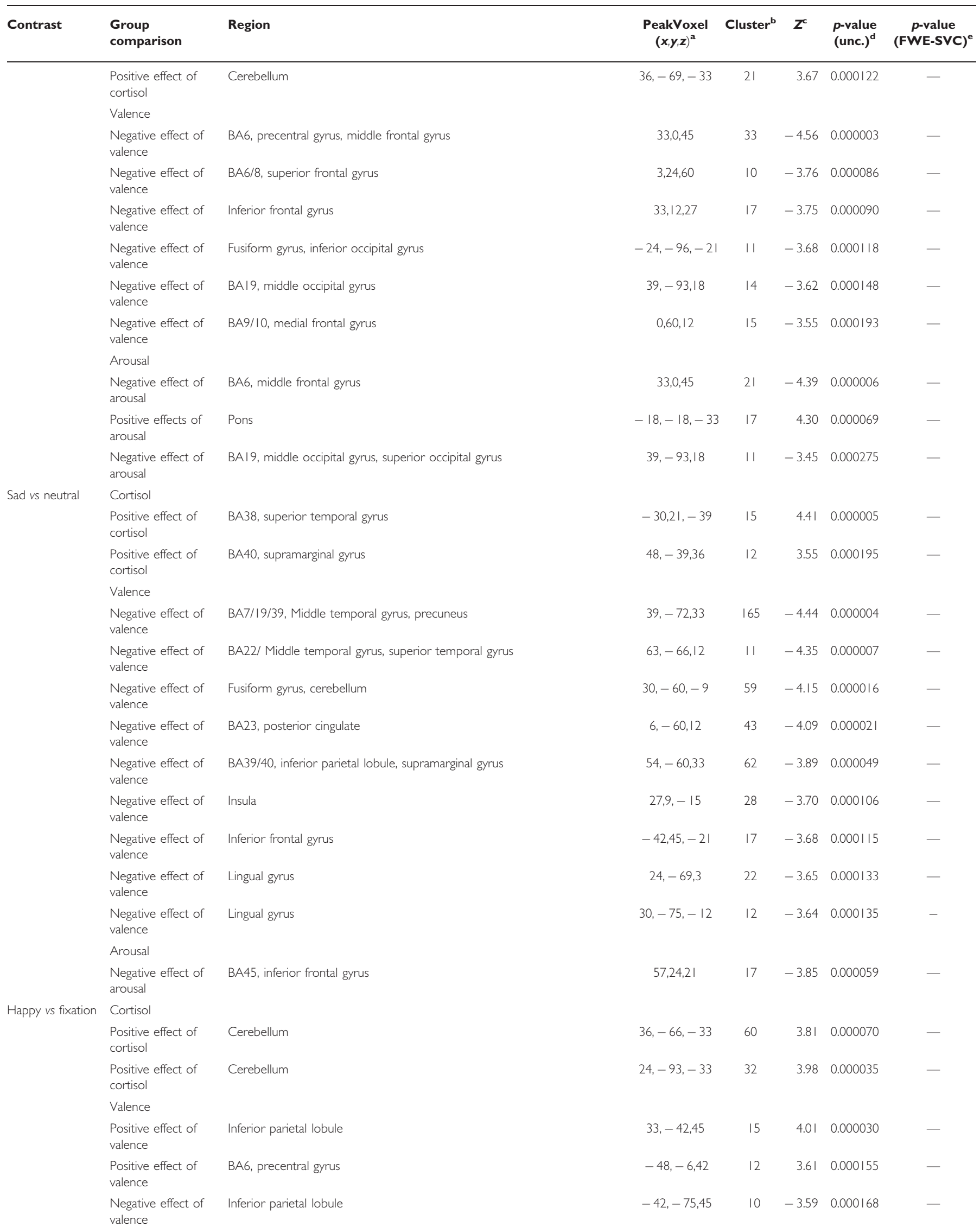


Table 2 (Continued)

\begin{tabular}{|c|c|c|c|c|c|c|c|}
\hline Contrast & $\begin{array}{l}\text { Group } \\
\text { comparison }\end{array}$ & Region & $\begin{array}{l}\text { PeakVoxel } \\
(x, y, z)^{\mathrm{a}}\end{array}$ & Cluster $^{\mathbf{b}}$ & $z^{c}$ & $\begin{array}{l}p \text {-value } \\
\text { (unc.) }^{\text {d }}\end{array}$ & $\begin{array}{c}p \text {-value } \\
\text { (FWE-SVC) }\end{array}$ \\
\hline & $\begin{array}{l}\text { Positive effect of } \\
\text { valence }\end{array}$ & BA6/44, precentral gyrus, inferior frontal gyrus & $60,3,15$ & 10 & 3.47 & 0.000256 & - \\
\hline & Arousal & & & & & & \\
\hline & $\begin{array}{l}\text { Positive effect of } \\
\text { arousal }\end{array}$ & Inferior parietal lobule & $-42,-72,45$ & 15 & 3.94 & 0.000041 & - \\
\hline & $\begin{array}{l}\text { Positive effect of } \\
\text { cortisol }\end{array}$ & Medial frontal gyrus & $-6,42,-15$ & 10 & 3.38 & 0.000361 & - \\
\hline & $\begin{array}{l}\text { Positive effect of } \\
\text { cortisol }\end{array}$ & BA34, subcallosal gyrus & $9,6,-15$ & 12 & 3.60 & 0.000160 & - \\
\hline & $\begin{array}{l}\text { Positive effect of } \\
\text { cortisol }\end{array}$ & BA22/42, superior temporal gyrus & $60,-9,6$ & 21 & 3.88 & 0.000052 & - \\
\hline & Valence & & & & & & \\
\hline & $\begin{array}{l}\text { Positive effect of } \\
\text { valence }\end{array}$ & Inferior frontal gyrus & $-54,9,3$ & 11 & 3.35 & 0.000407 & - \\
\hline & Arousal & & & & & & \\
\hline & - & No voxels survive & - & - & - & - & - \\
\hline \multicolumn{8}{|c|}{ Multiple regression on contrasts using faces only } \\
\hline \multirow{3}{*}{$\begin{array}{l}\text { Sad faces vs } \\
\text { fixation }\end{array}$} & Cortisol & & & & & & \\
\hline & - & No voxels survive & - & - & - & - & - \\
\hline & Valence & & & & & & \\
\hline & $\begin{array}{l}\text { Positive effect of } \\
\text { cortisol }\end{array}$ & BAII, middle frontal gyrus & $33,42,-9$ & 31 & 4.42 & 0.000005 & - \\
\hline & $\begin{array}{l}\text { Positive effect of } \\
\text { cortisol }\end{array}$ & BA6/9/44/45, inferior frontal gyrus & $-60,12,21$ & 234 & 4.40 & 0.000005 & - \\
\hline & $\begin{array}{l}\text { Positive effect of } \\
\text { cortisol }\end{array}$ & BAIO, superior frontal gyrus, medial frontal gyrus & $-15,66,6$ & 44 & 4.08 & 0.000023 & - \\
\hline & $\begin{array}{l}\text { Positive effect of } \\
\text { cortisol }\end{array}$ & BAII, superior frontal gyrus & $|5,5|,-2 \mid$ & 11 & 4.02 & 0.000029 & - \\
\hline & $\begin{array}{l}\text { Positive effect of } \\
\text { cortisol }\end{array}$ & Inferior parietal lobule, supramarginal gyrus & $54,-36,30$ & 17 & 3.73 & 0.000094 & - \\
\hline & $\begin{array}{l}\text { Positive effect of } \\
\text { cortisol }\end{array}$ & Superior frontal gyrus, medial frontal gyrus & $-21,42,24$ & 32 & 3.67 & 0.000120 & - \\
\hline & $\begin{array}{l}\text { Positive effect of } \\
\text { cortisol }\end{array}$ & Middle frontal gyrus & $-27,12,33$ & 11 & 3.55 & 0.000192 & - \\
\hline & $\begin{array}{l}\text { Positive effect of } \\
\text { cortisol }\end{array}$ & Inferior frontal gyrus & $-36,3,36$ & 12 & 3.44 & 0.000291 & - \\
\hline & Valence & & & & & & \\
\hline & - & No voxels survive & - & - & - & - & - \\
\hline & Arousal & & & & & & \\
\hline & $\begin{array}{l}\text { Positive effect of } \\
\text { arousal }\end{array}$ & BA20/21, middle temporal gyrus, inferior temporal gyrus & $-45,3,-36$ & 17 & 3.63 & 0.000140 & - \\
\hline & $\begin{array}{l}\text { Positive effect of } \\
\text { arousal }\end{array}$ & BA8, superior frontal gyrus & $18,39,54$ & 17 & 3.53 & 0.000205 & - \\
\hline
\end{tabular}


Table 2 (Continued)

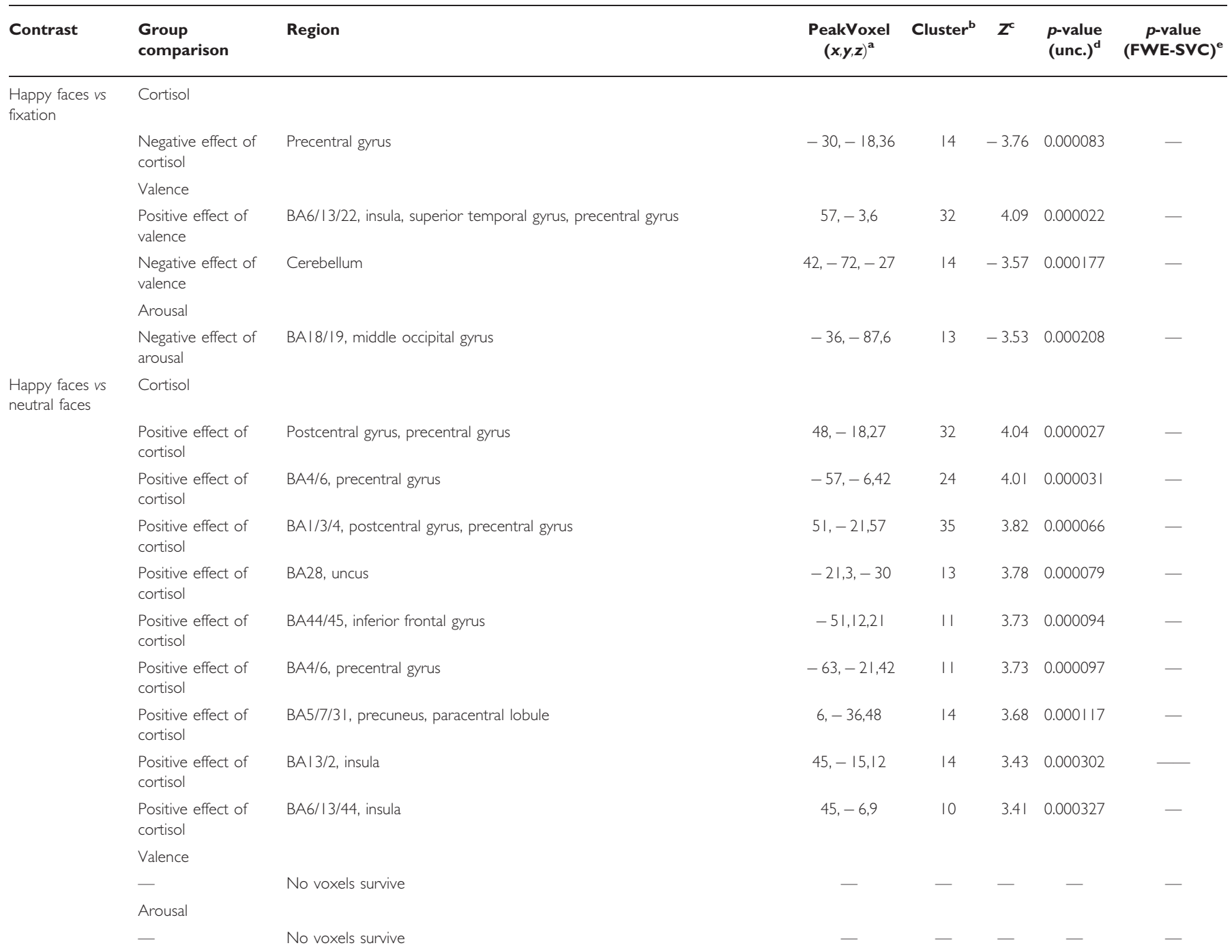

Multiple regression on contrasts using IAPS only

Sad IAPS vs Cortisol

fixation

\begin{tabular}{|c|c|}
\hline $\begin{array}{l}\text { Positive effect of } \\
\text { cortisol }\end{array}$ & Cerebellum \\
\hline $\begin{array}{l}\text { Positive effect of } \\
\text { cortisol }\end{array}$ & Cerebellum \\
\hline $\begin{array}{l}\text { Positive effect of } \\
\text { cortisol }\end{array}$ & Cerebellum \\
\hline \multicolumn{2}{|l|}{ Valence } \\
\hline $\begin{array}{l}\text { Negative effect of } \\
\text { valence }\end{array}$ & BAI9/30, culmen \\
\hline $\begin{array}{l}\text { Negative effect of } \\
\text { valence }\end{array}$ & BAI9, superior occipital gyrus \\
\hline $\begin{array}{l}\text { Negative effect of } \\
\text { valence }\end{array}$ & BA30, Posterior cingulate, cuneus \\
\hline $\begin{array}{l}\text { Negative effect of } \\
\text { valence }\end{array}$ & BA6, Middle frontal gyrus \\
\hline $\begin{array}{l}\text { Negative effect of } \\
\text { valence }\end{array}$ & BAI8/30, Lingual gyrus, posterior cingulate \\
\hline $\begin{array}{l}\text { Positive effect of } \\
\text { valence }\end{array}$ & BAI $8 / 19$, Cuneus \\
\hline Arousal & \\
\hline
\end{tabular}

$\begin{array}{cccc}27,-93,-30 & 15 & 4.00 & 0.000032 \\ 36,-69,-33 & 21 & 3.92 & 0.000044 \\ 24,-57,-27 & 12 & 3.69 & 0.000111 \\ & & & \\ -9,-45,-6 & 27 & 4.30 & 0.000008 \\ 39,-93,21 & 49 & 4.16 & 0.000016 \\ 21,-72,6 & 25 & -4.01 & 0.000030 \\ 33,0,45 & 24 & 3.99 & 0.000032 \\ -21,-60,3 & 30 & -3.79 & 0.000075 \\ 15,-96,21 & 12 & 3.64 & 0.000135\end{array}$

Arousal 
Table 2 (Continued)

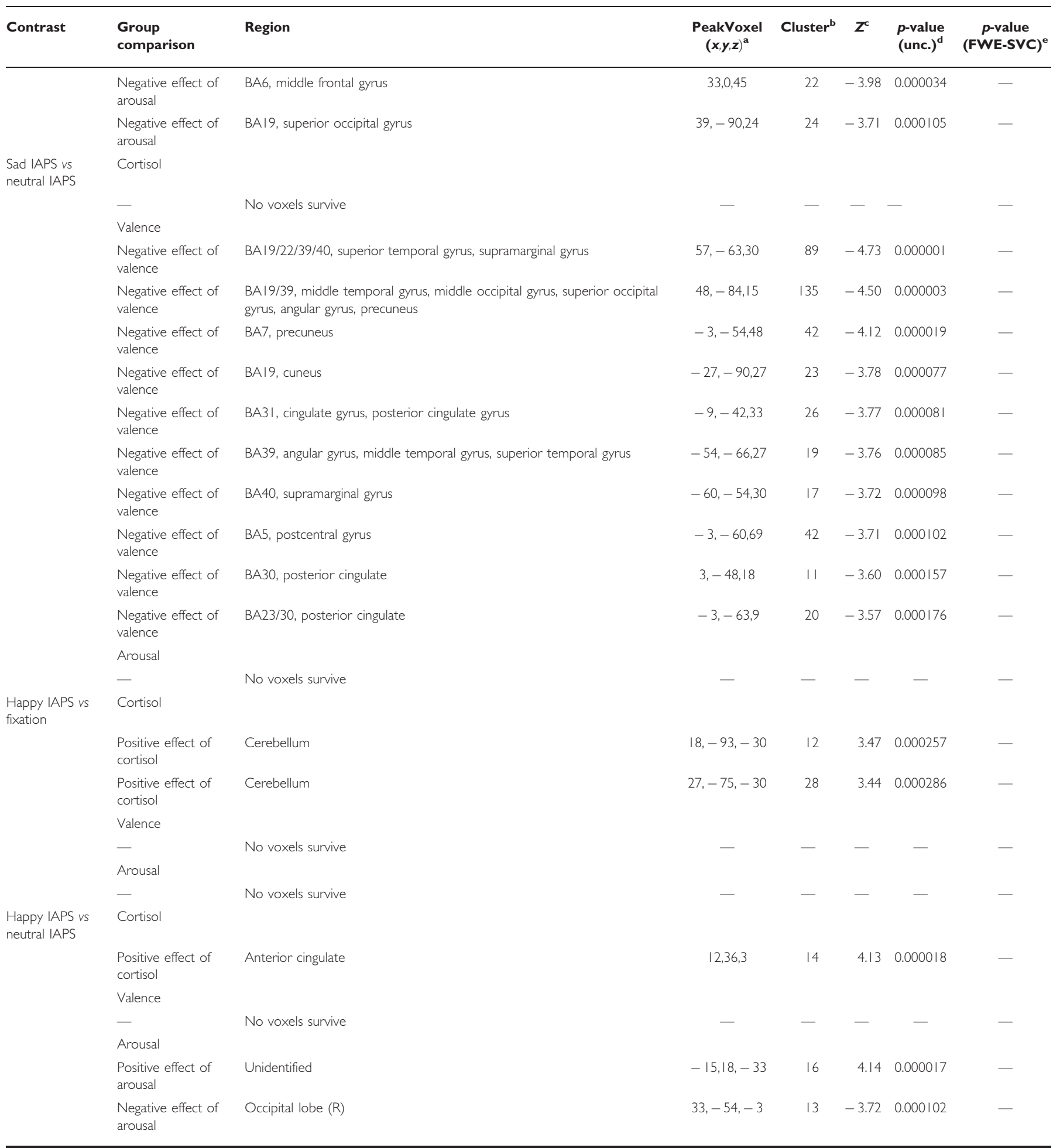

${ }^{a}$ Stereotactic coordinates from MNII 52 reference.

${ }^{\mathrm{b}}$ Cluster size in voxels.

'Negative $Z$-scores indicate a reduced activation of the hydrocortisone group relative to the placebo group.

${ }^{\mathrm{d} A l l}$ foci meet min threshold of $p<0.00 \mathrm{I}$, uncorrected with an extend threshold 10 voxels.

esignificant $p$-values after small volume correction.

*Foci are significant at a family-wise error corrected threshold of $p<0.05$ after small volume correction. $p$-values listed reflect the peak voxel within a cluster. 

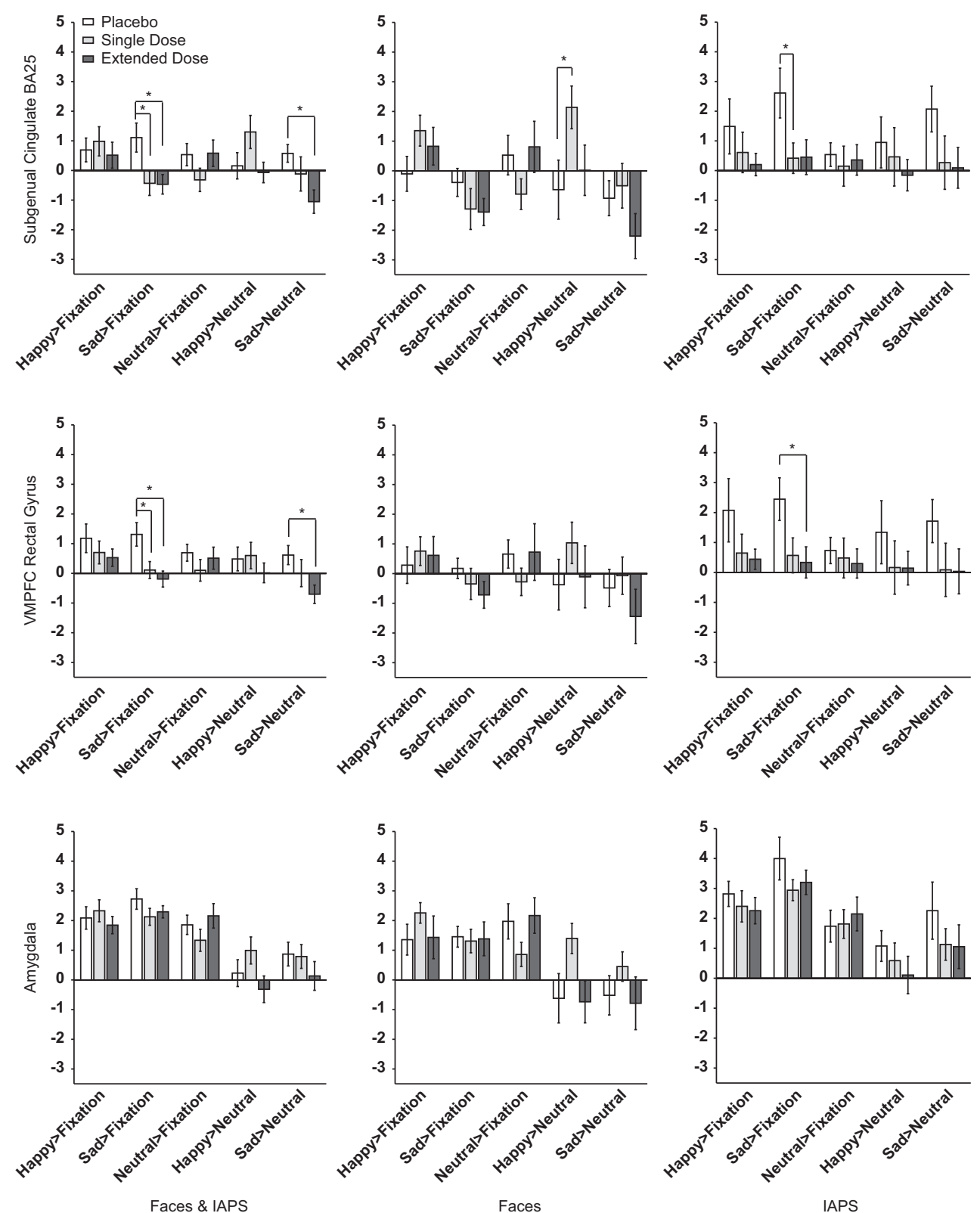

Figure I Regions of interest extractions from the subgenual cingulate (panel I) ventral medial prefrontal cortex (panel 2), and the amygdala (panel 3). Significant group differences were detected for contrasts involving sad stimuli in the subgenual cingulate and the ventral medial prefrontal cortex (VMPFC). * $p<0.05$ after Bonferroni correction.

\section{Blinding}

The majority of subjects $(42 / 61,69 \%)$ reported that they thought they received placebo, including those that received HCT (26/41, 63\%). Overall, the groups did not significantly differ in their guesses $\chi^{2}(4)=5.136, p=0.274$ regarding their assigned dose regimen. There were also no significant differences in guessing patterns among just the HCT groups $\chi^{2}(2)=0.148, p=0.929$.

\section{Subjective Emotional Reactions to Images and Mood}

Across all groups the images selected as happy and sad stimuli successfully induced moderate feelings of the target emotions. Happy stimuli induced moderate feelings of happiness and sad stimuli induced moderate feelings of sadness (Figure 3). There was a significant omnibus effect of group on arousal ratings of sad stimuli but no effects for other stimuli types. The group effect on sad stimuli was driven by greater arousal in the ED relative to the placebo group. This effect was observed when all sad stimuli are pooled or when sad IAPS are considered alone (Figure 3). When sad faces are considered alone this effect of HCT on arousal is similar qualitatively but not statistically significant. We tested for emotion type (happy, sad, neutral) by group interactions on arousal ratings to formally determine if these effects were specific to sad stimuli. The interaction was not significant when IAPS stimuli and faces are pooled 
Table 3 Subjective Emotional Reactions to Experimental Stimuli, PANAS Mood Measures Over 4 Days of HCT Exposure, and HCTInduced Elevations in Salivary Cortisol Levels

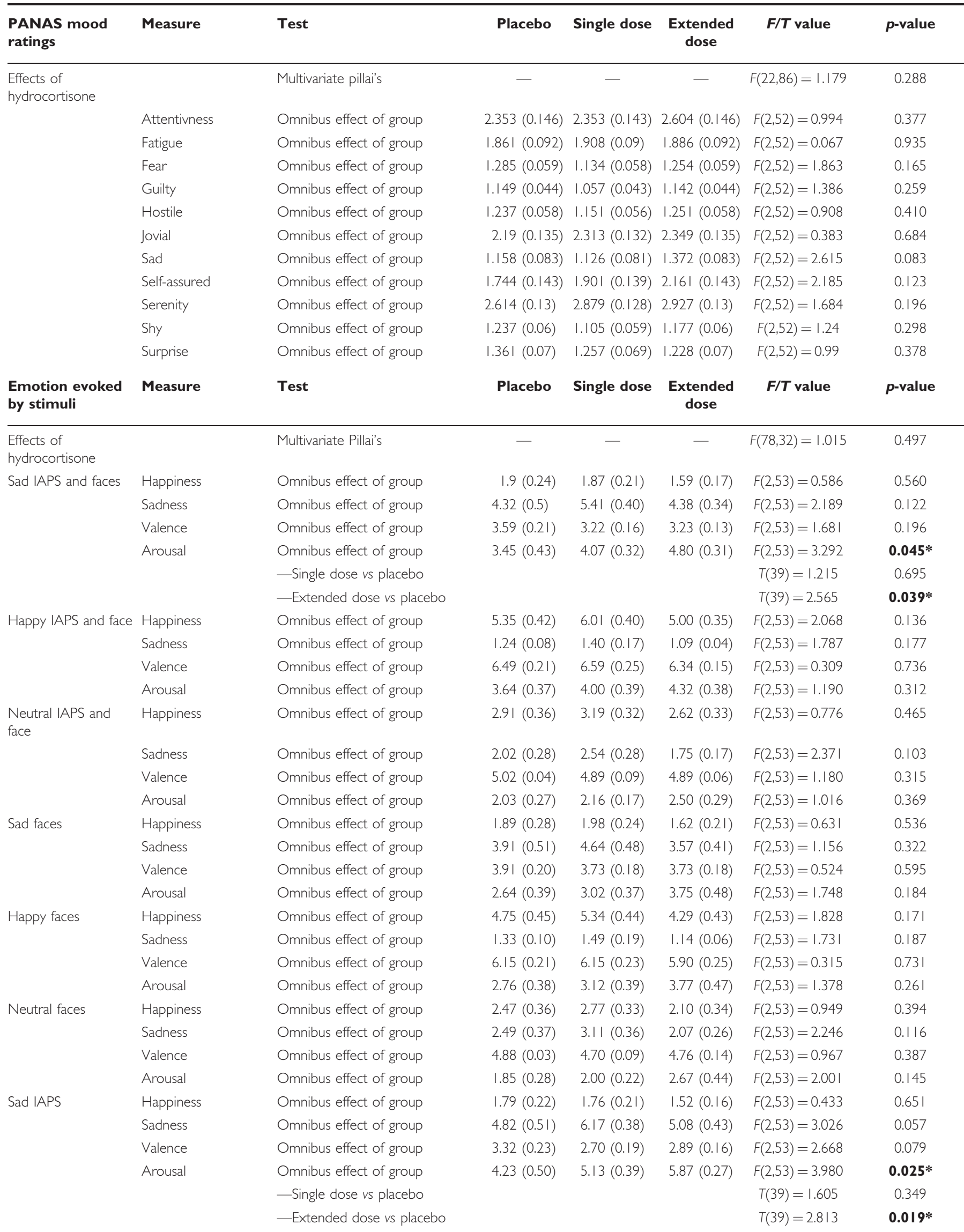


Table 3 (Continued)

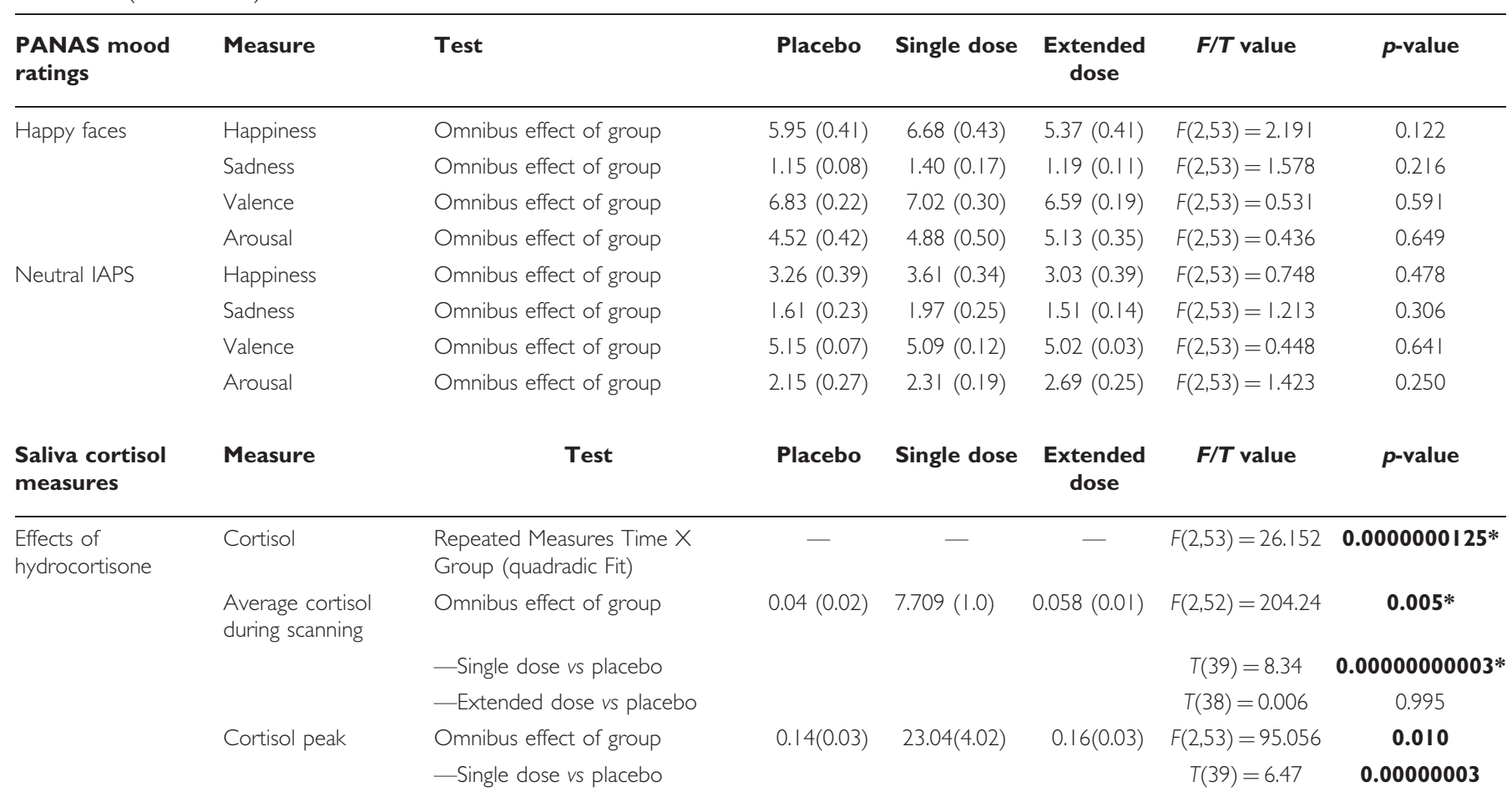

Extended Dose vs. Placebo T(38)=0.018 0.996. Subjective arousal was only significantly increased in reaction to viewing sad stimuli in the extended dose group. Similar non-significant effects of hydrocortisone on arousal were also seen on other types of stimuli (see also Figure 3 and Figure 4).

*Bold value indicate $p<0.05$ for omnibus tests or after Bonferroni correction.

Pillai's trace $\mathrm{F}(4,106)=1.430, p=0.229)$ nor when faces $\mathrm{F}(4,106)=0.267, \quad p=0.899) \quad$ or IAPS $\mathrm{F}(4,106)=1.623$, $p=0.174)$ are considered separately. We observed no other effects of HCT on ratings of any other stimulus type.

HCT administered over 4 days did not alter mood states as measured by any of the 60 adjectives or the 11 PANAS-X subscales for the either of the HCT groups compared with the $\mathrm{P}$ group (Table 3 ).

\section{Saliva Cortisol Levels}

Cortisol levels measured in saliva were elevated in the SD group after taking HCT. The pattern of cortisol observed in the SD group fit a quadratic pattern of HCT absorption and then metabolism. There were highly significant group differences for this pattern (Table 3). The placebo and the ED maintained much lower and unchanging levels of salivary cortisol over the course of fMRI scanning with no significant differences between them. The peak levels of salivary cortisol and the average salivary cortisol levels during fMRI scanning were highly elevated in the SD group, but not in the ED or P groups (Table 3 ).

\section{DISCUSSION}

We examined the effects of a SD and a 4-day administration of HCT on activity in sgACC, VMPFC, and amygdala in response to viewing happy, sad, and neutral emotional stimuli. HCT administration reduced sgACC and VMPFC activations evoked by sad stimuli. HCT administration had few other consistent effects on activity evoked by other emotions or in other brain regions. We also found that 4-day exposure to HCT increased the subjective arousal participants report after viewing sad stimuli but did not affect general mood ratings.

Previous neuroimaging studies have found that exogenous glucocorticoid administration can increase medial temporal lobe (De Quervain et al, 2003) and hippocampus (Abercrombie et al, 2011) activity, and decrease amygdala activity and connectivity with the VMPFC (Henckens et al, 2010; Lovallo et al, 2010), though none of these previous studies specifically addressed sadness-related emotion activity. In contrast, our study focused explicitly on emotional processes and brain regions implicated in MDD, including sadness. Both sgACC hyperactivity (Mayberg et al, 1997, 1999) and decreased brain sensitivity to cortisol signaling (glucocorticoid resistance) have been associated with the symptoms in MDD (Holsboer, 2000; Pariante, 2006). Our results suggest a mechanism that could link these two findings. If cortisol functions to reduce sgACC activity in response to sad stimuli in a healthy state, as we show here, then the development of glucocorticoid resistance in MDD may disinhibit sadness-induced sgACC activity. This disinhibition would then create the sgACC hyperactivity that has been observed in MDD patients relative to healthy subjects. These findings fit well within the predictions of an overarching corticosteroid receptor hypothesis of depression (Holsboer, 2000). Our study extends this theory and predicts that hyperactivity of the 

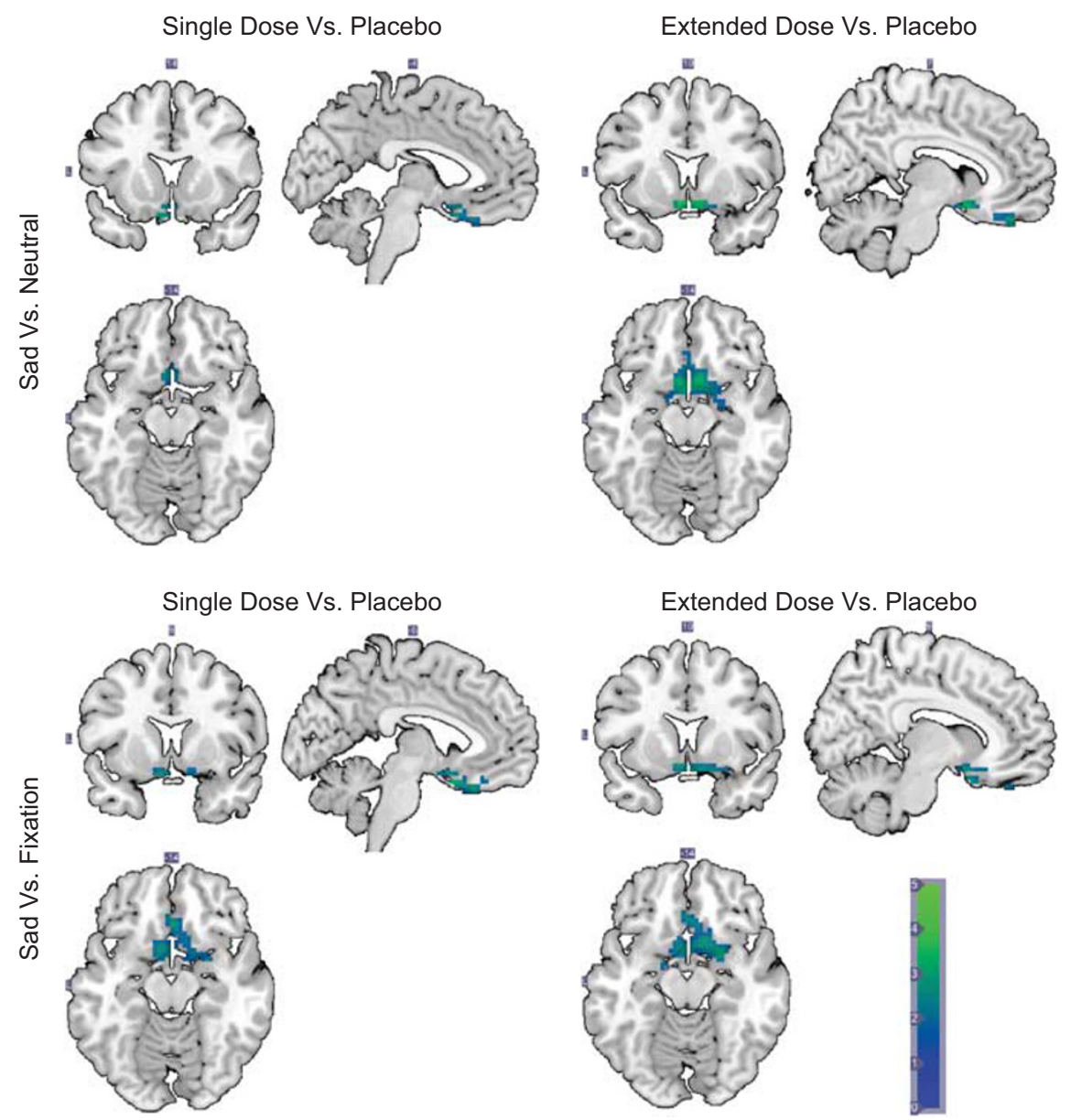

Figure 2 Decreased activation of the subgenual cingulate cortex in groups receiving hydrocortisone (HCT) on contrasts involving sad stimuli. Qualitatively similar effects were seen in both HCT groups. Voxel-wise statistical tests at $p<0.05$ after family-wise error and small volume correction are only significant for peak voxels in the extended dose group on the sad vs neutral contrast (upper-right panel). The maps above are subject to a $p<0.05$ threshold and a cluster threshold of five voxels for qualitative display purposes. See Table 2 for statistics.

sgACC should occur subsequent to glucocorticoid receptor downregulations in the development of MDD. We also predict that pharmacological treatments that increase glucocorticoid receptors in the sgACC should also decrease sgACC hyperactivity.

It is noteworthy that we observe similarly decreased sgACC after both acute and 4-day HCT administration. This finding supports the idea that sgACC activity is sensitive to glucocorticoids, but raises questions about the mechanisms involved, as saliva cortisol levels were very different at the time of scanning in the two groups. One explanation for these similarities could be that the effects on the brain are driven by a recent acute rise in glucocorticoid levels. At the time of scanning, both HCT regimens had a recent rise in cortisol levels. The ED subject took the final dose of their HCT regimen $8 \mathrm{~h}$ before scanning. Although it was metabolized by the time of the scanning, it may be producing effects similar to the dose administered to the SD subjects, taken $2 \mathrm{~h}$ before scanning. The effects of glucocorticoids can have a rapid onset and produce effects that outlast the elevated circulating glucocorticoids that caused them. Such fast and long-lasting effects of glucocorticoids have previously been demonstrated in animal studies (Rose, 2000) and may be mediated through fast-acting membrane-bound corticosteroid receptors (Orchinik et al, 1991).

HCT administered over 4 days increased arousal experienced when viewing sad stimuli, but did not affect general PANAS mood ratings. Previous work has demonstrated that in healthy subjects glucocorticoid administration can improve memory for arousing stimuli (Buchanan and Lovallo, 2001). However, one recent study has shown no effect of glucocorticoids on arousal (Abercrombie et al, 2011). This recent finding was based on general mood measures of arousal (PANAS), where we also find no effects of HCT. The arousal enhancing effect of HCT in our study was only observed when participants were reporting arousal that was evoked by a sad stimulus, as opposed to rating their generally aroused mood. Our results suggest that HCT may also be increasing arousal to other stimuli as well, although to a lesser extent (Figure 3). By increasing arousal evoked from sad stimuli to a greater degree than other types of stimuli glucocorticoids could be generating a temporary bias toward attending to negative emotional stimuli in healthy subjects. As high levels of glucocorticoid often occur during times of high physiological or psychological stress, such a bias may be appropriate in many cases. However, additional studies are needed to determine how 

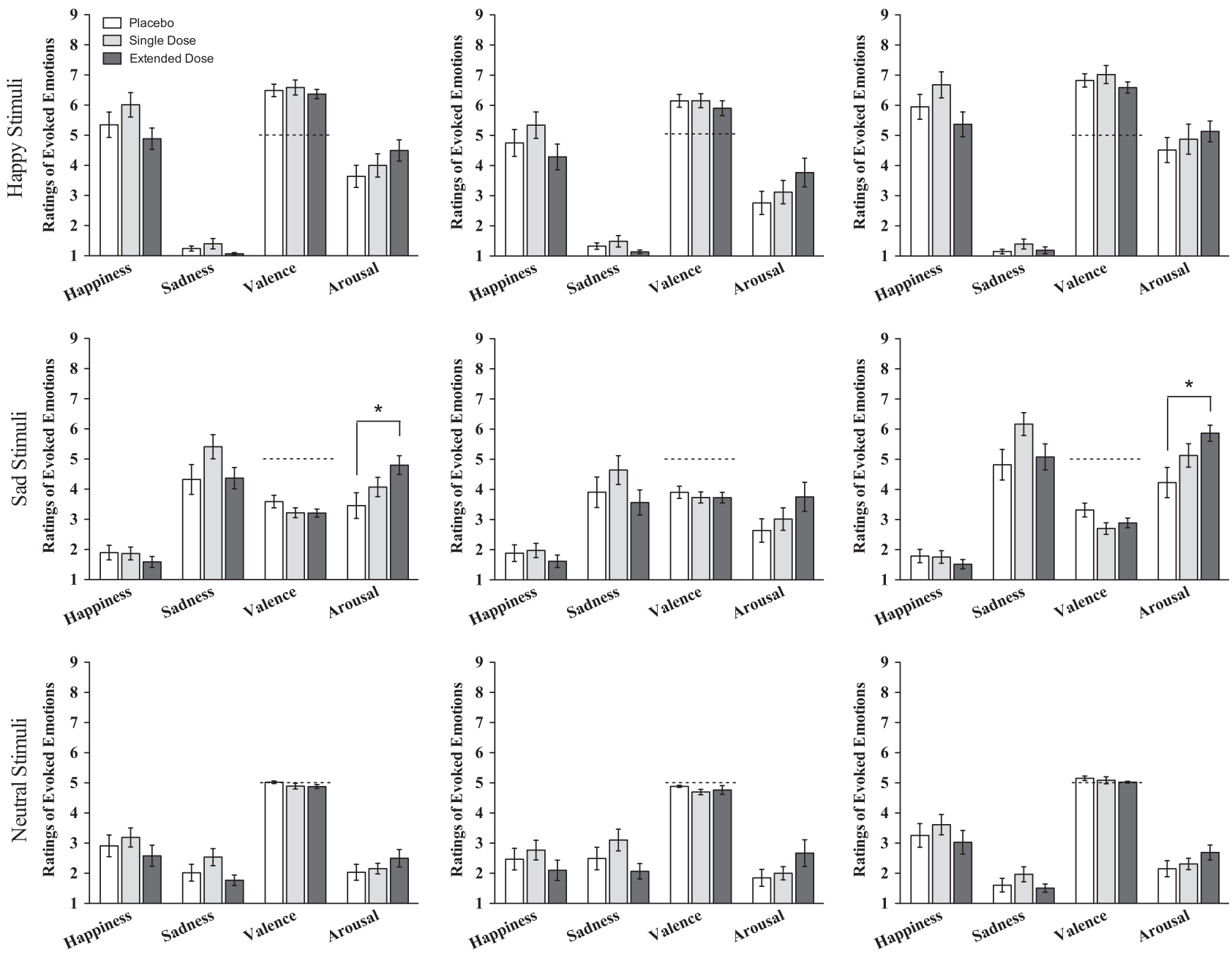

Faces \& IAPS

Faces

IAPS

Figure 3 Subjective ratings are displayed for each type of experimental stimuli. The first column displays data for the each kind of emotional stimuli (happy, sad, neutral). The second and third column displays the same data when only faces or only International Affective Picture System (IAPS) stimuli are considered. The dashed line above the valence measure denotes the neutral valence rating. Stimuli ratings above 5 indicate a positive valence and below 5 indicate a negative valence. In the hydrocortisone groups, the ratings of arousal across all stimuli are elevated. However, this elevation in arousal only achieves statistical significance for sad stimuli rated by the extended dose group. $* p<0.05$ after Bonferroni correction.

cortisol effects arousal across its physiological range. These findings may also fit predictions made by the corticosteroid receptor hypothesis of depression (Holsboer, 2000). If one of cortisol's normal physiological functions is to increase arousal, then a reduced sensitivity to cortisol in depression could result in more vegetative symptoms and psychomotor slowing, particularly in response to evocative stimuli. Low-arousal symptoms are commonly found in melancholic depression subtypes, which also have amongt the most reliable HPA dysregulation (Stetler and Miller, 2011).

Our results confirm previous findings (Britton et al, 2005) that IAPS stimuli are generally more emotionally evocative than faces (Figure 3). Correspondingly, the sgACC and VMPF showed more robust activations when sad IAPS were being viewed as compared with sad faces. However, our multiple regression models failed to show a direct linear relationship between the strength of these activations and the valence/arousal ratings of either faces or IAPS pictures. Nevertheless, future studies investigating sgACC or VMPFC sensitivity to HCT may consider choosing more evocative stimulus sets to maximize the contrast with HCT-induced suppression in these regions.

One limitation of our study is that the dose of HCT that we used in the SD group achieved salivary cortisol concentrations that were in excess of most normal physiological stress responses. Although this is an important consideration, it is worth noting that receptor binding is the limiting factor of the physiological effects of HCT. Therefore, the high concentrations generated in our study may not result in exaggerated physiological effects as receptor binding has a limited capacity. The similar results we observed among the SD and ED groups seem to support the notion that our higher dose is not generating non-physiological effects.

As a manipulation check, we analyzed which brain regions responded to sad stimuli in healthy controls. We observed typical visual-emotion-related patterns (activation in the visual cortex, thalamus, superior colliculus, amygdala, hippocampus, caudate, putamen, dorsal medial prefrontal cortex, dorsal lateral prefrontal cortex, sgACC, VMPFC, and deactivation in the superior temporal gyrus, insula, anterior cingulate, dorsal lateral prefrontal cortex, and 

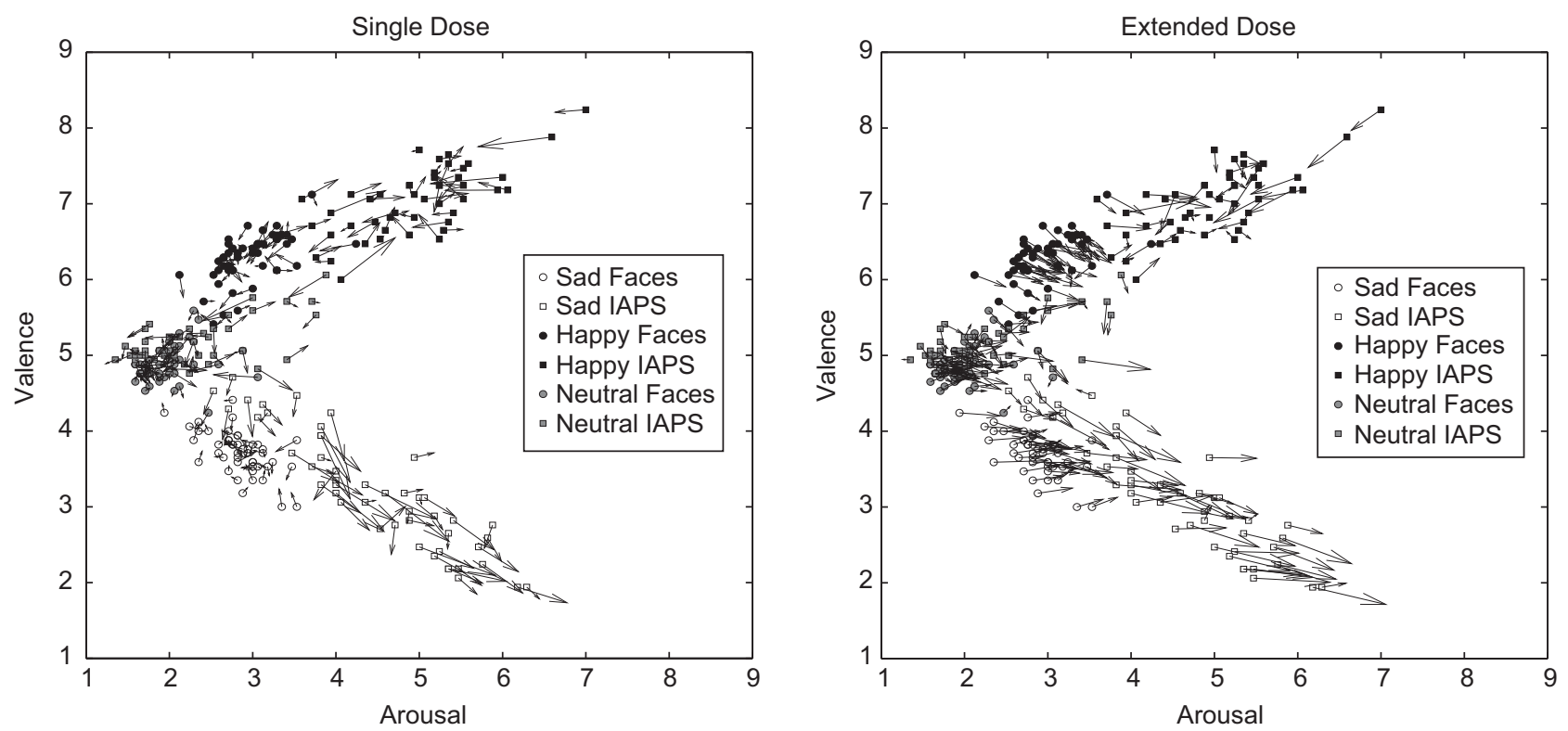

Figure 4 Subjective ratings of each of the experimental stimuli are plotted in valence/arousal space for the placebo group. Arrows indicate the vector of shifts toward increasing arousal in the hydrocortisone groups. The left panel quiver plot demonstrates placebo group to single-dose group change vectors. The right panel demonstrates placebo group to extended dose group change vectors. In the extended dose group, the most prominent shifts are in the stimuli occupying the low valence portions of the valence/arousal space, which correspond to sad stimuli. IAPS, International Affective Picture System.

posterior cingulate/precuneus (data not shown), when applying the false-discovery rate correction for multiple comparisons $(p<0.05, k=10)$.

In conclusion, we present evidence suggesting that HCT decreases the sgACC and VMPFC activity evoked by sad stimuli. We also demonstrate that HCT increases the subjective arousal experienced while viewing sad stimuli. These findings may have important implications for research on the pathophysiology of MDD, as this is the first study to demonstrate that elevated cortisol can cause functional decreases in activation in the sgACC during sad conditions. This finding suggests a plausible theory of glucocorticoid resistance causing sgACC hyperactivity in MDD.

\section{ACKNOWLEDGEMENTS}

This work was funded by the National Institutes of Health F31MH073223, R24MH075999, and the University of Michigan Depression Center Rachel Upjohn clinical scholar award.

\section{DISCLOSURE}

Dr Taylor has received research support from St Jude Medical and Neuronetics. All the other authors declare no conflict of interest.

\section{REFERENCES}

Abercrombie HC, Jahn AL, Davidson RJ, Kern S, Kirschbaum C, Halverson J (2011). Cortisol's effects on hippocampal activation in depressed patients are related to alterations in memory formation. J Psychiatr Res 45: 15-23.
Britton JC, Taylor SF, Sudheimer KD, Liberzon I (2005). Facial expressions and complex IAPS pictures: common and differential networks. Neuroimage 31: 906-919.

Brown ES, Woolston DJ, Frol A, Bobadilla L, Khan DA, Hanczyc M et al (2004). Hippocampal volume, spectroscopy, cognition, and mood in patients receiving corticosteroid therapy. Biol Psychiatry 55: 538-545.

Brown ES, Woolston DJ, Frol AB (2008). Amygdala volume in patients receiving chronic corticosteroid therapy. Biol Psychiatry 63: 705-709.

Buchanan TW, Lovallo WR (2001). Enhanced memory for emotional material following stress-level cortisol treatment in humans. Psychoneuroendocrinology 26: 307-317.

Cho K, Little HJ (1999). Effects of corticosterone on excitatory amino acid responses in dopamine-sensitive neurons in the ventral tegmental area. Neuroscience 88: 837-845.

Damasio H (2005). Human Brain Anatomy in Computerized Images. 2nd edn. Oxford University Press: New York, NY, xvp 540.

De Kloet ER, Vreugdenhil E, Oitzl MS, Joels M (1998). Brain corticosteroid receptor balance in health and disease. Endocr Rev 19: 269-301.

De Quervain DJ, Henke K, Aerni A, Treyer V, McGaugh JL, Berthold $\mathrm{T}$ et al (2003). Glucocorticoid-induced impairment of declarative memory retrieval is associated with reduced blood flow in the medial temporal lobe. Eur J Neurosci 17: 1296-1302.

Greden JF, Albala AA, Haskett RF, James NM, Goodman L, Steiner $M$ et al (1980). Normalization of dexamethasone suppression test: a laboratory index of recovery from endogenous depression. Biol Psychiatry 15: 449-458.

Gur RC, Schroeder L, Turner T, McGrath C, Chan RM, Turetsky BI et al (2002). Brain activation during facial emotion processing. Neuroimage 16(3 Part 1): 651-662.

Henckens MJ, van Wingen GA, Joels M, Fernandez G (2010). Timedependent effects of corticosteroids on human amygdala processing. J Neurosci 30: 12725-12732.

Holsboer F (2000). The corticosteroid receptor hypothesis of depression. Neuropsychopharmacology 23: 477-501. 
Imfeld A. http://www.aimfeld.ch/neurotools/neurotools.html.

Karst H, Nair S, Velzing E, Rumpff-van Essen L, Slagter E, Shinnick-Gallagher P et al (2002). Glucocorticoids alter calcium conductances and calcium channel subunit expression in basolateral amygdala neurons. Eur J Neurosci 16: 1083-1089.

Lang PJ, Bradley MM, Cuthbert BN (1999). International Affective Picture System (IAPS): Instruction Manual and Affective Ratings.

Ling MH, Perry PJ, Tsuang MT (1981). Side effects of corticosteroid therapy. Psychiatric aspects. Arch Gen Psychiatry 38: 471-477.

Lovallo WR, Robinson JL, Glahn DC, Fox PT (2010). Acute effects of hydrocortisone on the human brain: an fMRI study. Psychoneuroendocrinology 35: 15-20.

Mai JK, Assheuer J, Paxinos G (2004). Atlas of the Human Brain. 2nd edn. Elsevier Academic Press: Amsterdam, Boston, viii, pp 246.

Mayberg HS, Brannan SK, Mahurin RK, Jerabek PA, Brickman JS, Tekell JL et al (1997). Cingulate function in depression: a potential predictor of treatment response. Neuroreport 8: 1057-1061.

Mayberg HS, Liotti M, Brannan SK, McGinnis S, Mahurin RK, Jerabek PA et al (1999). Reciprocal limbic-cortical function and negative mood: converging PET findings in depression and normal sadness. Am J Psychiatry 156: 675-682.

Mitra R, Sapolsky RM (2008). Acute corticosterone treatment is sufficient to induce anxiety and amygdaloid dendritic hypertrophy. Proc Natl Acad Sci USA 105: 5573-5578.

Orchinik M, Murray TF, Moore FL (1991). A corticosteroid receptor in neuronal membranes. Science 252: 1848-1851.

Pariante CM (2006). The glucocorticoid receptor: part of the solution or part of the problem? J Psychopharmacol 20(4 Suppl): 79-84.

Pariante CM, Thomas SA, Lovestone S, Makoff A, Kerwin RW (2004). Do antidepressants regulate how cortisol affects the brain? Psychoneuroendocrinology 29: 423-447.

Reuter M (2002). Impact of cortisol on emotions under stress and nonstress conditions: a pharmacopsychological approach. Neuropsychobiology 46: 41-48.

Rose JD (2000). Corticosteroid actions from neuronal membrane to behavior: neurophysiological mechanisms underlying rapid behavioral effects of corticosterone. Biochem Cell Biol 78: 307-315.

Sapolsky RM (2000). Glucocorticoids and hippocampal atrophy in neuropsychiatric disorders. Arch Gen Psychiatry 57: 925-935.

Sarrieau A, Dussaillant M, Agid F, Philibert D, Agid Y, Rostene W (1986). Autoradiographic localization of glucocorticosteroid and progesterone binding sites in the human post-mortem brain. J Steroid Biochem 25: 717-721.

Sheehan DV, Janavs J, Baker R, Harnett-Sheehan K, Knapp E, Sheehan M et al (1998). MINI - Mini International Neuropsychiatric Interview - English Version 5.0.0 - DSM-IV. J Clin Psychiatry 59: 34-57.
Stetler C, Miller GE (2011). Depression and hypothalamicpituitary-adrenal activation: a quantitative summary of four decades of research. Psychosom Med 73: 114-126.

Sudheimer K, Winn B, Kerndt G, Shoaps J, Davis K, Fobbs A et al (2002) www.msu.edu/ brains/brains/human/.

Tzourio-Mazoyer N, Landeau B, Papathanassiou D, Crivello F, Etard O, Delcroix N et al (2002). Automated anatomical labeling of activations in SPM using a macroscopic anatomical parcellation of the MNI MRI single-subject brain. Neuroimage 15: 273-289.

Wada K, Yamada N, Sato T, Suzuki H, Miki M, Lee Y et al (2001). Corticosteroid-induced psychotic and mood disorders: diagnosis defined by DSM-IV and clinical pictures. Psychosomatics 42: 461-466.

Wada K, Yamada N, Suzuki H, Lee Y, Kuroda S (2000). Recurrent cases of corticosteroid-induced mood disorder: clinical characteristics and treatment. J Clin Psychiatry 61: 261-267.

Watson D, Clark LA (1994). http://www.psychology.uiowa.edu/ faculty/watson/PANAS-X.pdf.

Watson D, Clark LA, Tellegen A (1988). Development and Validation of Brief Measures of Positive and Negative Affect the Panas Scales. J Pers Soc Psychol 54: 1063-1070.

Watzka M, Beyenburg S, Blumcke I, Elger CE, Bidlingmaier F, Stoffel-Wagner B (2000a). Expression of mineralocorticoid and glucocorticoid receptor mRNA in the human hippocampus. Neurosci Lett 290: 121-124.

Watzka M, Bidlingmaier F, Beyenburg S, Henke RT, Clusmann H, Elger CE et al (2000b). Corticosteroid receptor mRNA expression in the brains of patients with epilepsy. Steroids 65: 895-901.

Webster MJ, Knable MB, O'Grady J, Orthmann J, Weickert CS (2002). Regional specificity of brain glucocorticoid receptor mRNA alterations in subjects with schizophrenia and mood disorders. Mol Psychiatry 7: 985-994 924.

Wellman CL (2001). Dendritic reorganization in pyramidal neurons in medial prefrontal cortex after chronic corticosterone administration. J Neurobiol 49: 245-253.

Wirth MM, Scherer SM, Hoks RM, Abercrombie HC (2011). The effect of cortisol on emotional responses depends on order of cortisol and placebo administration in a within-subject design. Psychoneuroendocrinology.

Woolley CS, Gould E, McEwen BS (1990). Exposure to excess glucocorticoids alters dendritic morphology of adult hippocampal pyramidal neurons. Brain Res 531: 225-231.

Yang Y, Gu H, Zhan W, Xu S, Silbersweig DA, Stern E (2002). Simultaneous perfusion and BOLD imaging using reverse spiral scanning at 3T: characterization of functional contrast and susceptibility artifacts. Magn Reson Med 48: 278-289.

Zobel AW, Nickel T, Sonntag A, Uhr M, Holsboer F, Ising M (2001). Cortisol response in the combined dexamethasone/CRH test as predictor of relapse in patients with remitted depression. a prospective study. J Psychiatr Res 35: 83-94.

Zobel AW, Yassouridis A, Frieboes RM, Holsboer F (1999). Prediction of medium-term outcome by cortisol response to the combined dexamethasone-CRH test in patients with remitted depression. Am J Psychiatry 156: 949-951. 